\title{
A Study of Impulsive Multiterm Fractional Differential Equations with Single and Multiple Base Points and Applications
}

\author{
Yuji Liu' ${ }^{1}$ and Bashir Ahmad ${ }^{2}$ \\ ${ }^{1}$ Department of Mathematics, Guangdong University of Business Studies, Guangzhou 510320, China \\ ${ }^{2}$ Department of Mathematics, Faculty of Science, King Abdulaziz University, P.O. Box 80203, \\ Jeddah 21589, Saudi Arabia
}

Correspondence should be addressed to Bashir Ahmad; bashirahmad_qau@yahoo.com

Received 10 October 2013; Accepted 17 November 2013; Published 21 January 2014

Academic Editors: A. M. A. El-Sayed, A. Kılıçman, and S. C. O. Noutchie

Copyright (C) 2014 Y. Liu and B. Ahmad. This is an open access article distributed under the Creative Commons Attribution License, which permits unrestricted use, distribution, and reproduction in any medium, provided the original work is properly cited.

\begin{abstract}
We discuss the existence and uniqueness of solutions for initial value problems of nonlinear singular multiterm impulsive Caputo type fractional differential equations on the half line. Our study includes the cases for a single base point fractional differential equation as well as multiple base points fractional differential equation. The asymptotic behavior of solutions for the problems is also investigated. We demonstrate the utility of our work by applying the main results to fractional-order logistic models.
\end{abstract}

\section{Introduction}

The theory of impulsive differential equations describes processes which experience a sudden change of their state at certain moments. Processes with such a characteristic arise naturally and are often, for example, studied in physics, chemical technology, population dynamics, biotechnology, and economics. These processes are modeled by impulsive differential equations. In 1960, Milman and Myshkis introduced the concept of impulsive differential equations [1]. Afterwards, this subject was extensively investigated and several monographs have been published by many authors like Samoilenko and Perestyuk [2], Lakshmikantham et al. [3], Baino and Simeonov [4], Baino and Covachev [5], and Benchohra et al. [6].

Fractional differential equations (FDEs for short), regarded as the generalizations of ordinary differential equations to an arbitrary noninteger order, find their genesis in the work of Newton and Leibniz in the seventieth century. Recent investigations indicate that many physical systems can be modeled more accurately with the help of fractional derivatives [7]. Fractional differential equations, therefore, find numerous applications in the field of viscoelasticity, feedback amplifiers, electrical circuits, electroanalytical chemistry, fractional multipoles, and neuron modelling encompassing different branches of physics, chemistry, and biological sciences [8-10].

Some recent work on the existence of solutions for initial value problems of Caputo type impulsive fractional differential equations can be found in a series of papers [11-16], whereas the solvability of boundary value problems of impulsive differential equations involving Caputo fractional derivatives was investigated in [17-26].

In the left and right fractional derivatives $D_{a^{+}}^{\alpha} x$ and $D_{b^{-}}^{\alpha} x$, $a$ is called a left base point and $b$ right base point. Both $a$ and $b$ are called base points of fractional derivatives. A fractional differential equation (FDE) containing more than one base points is called a multiple base points FDE while an FDE containing only one base point is called a single base point FDE.

Henderson and Ouahab [12] studied the solvability of the following initial value problems for impulsive fractional differential equations:

$$
\begin{gathered}
D_{*}^{\alpha} u(t)=f(t, u(t)), \quad t \in(0, b] \backslash\left\{t_{1}, \ldots, t_{m}\right\}, \alpha \in(1,2], \\
u\left(t_{k}^{+}\right)=I_{k}\left(u\left(t_{k}^{-}\right)\right), \quad k=1,2, \ldots, m, \\
u^{\prime}\left(t_{k}^{+}\right)=J_{k}\left(u\left(t_{k}^{-}\right)\right), \quad k=1,2, \ldots, m, \\
u(0)=a, \quad u^{\prime}(0)=c,
\end{gathered}
$$




$$
\begin{gathered}
D_{*}^{\alpha} u(t)=f(t, u(t)), \quad t \in(0, b] \backslash\left\{t_{1}, \ldots, t_{m}\right\}, \alpha \in(0,1], \\
\Delta u\left(t_{k}\right)=u\left(t_{k}^{+}\right)-u\left(t_{k}^{-}\right)=I_{k}\left(y\left(t_{k}^{-}\right)\right), \quad k=1,2, \ldots, m, \\
u(0)=a,
\end{gathered}
$$

where $0<t_{1}<t_{2}<\cdots<t_{m}<b, b>0$ is a fixed real number, $f:[0, b] \times R \rightarrow R$ is continuous, $I_{k}, J_{k}: R \rightarrow R(k=1$, $2, \ldots, m)$ are continuous functions, $u\left(t_{k}^{+}\right)=\lim _{t \rightarrow t_{k}^{+}} u(t)$ and $u\left(t_{k}^{-}\right)=\lim _{t \rightarrow t_{k}^{-}} u(t)$ and $u^{\prime}\left(t_{k}^{+}\right)=\lim _{t \rightarrow t_{k}^{+}} u^{\prime}(t)$. One can see that both fractional differential equations in (1) are multiple base points FDEs with base points $0, t_{1}, t_{2}, \ldots, t_{m}$, which are in fact the impulse points.

In [27], the authors used the concept of upper and lower solutions together with Schauder's fixed point theorem to study the impulsive fractional-order differential equation:

$$
\begin{gathered}
{ }^{c} D^{\alpha} u(t)=f(t, u(t)), \quad t \in(0, b] \backslash\left\{t_{1}, \ldots, t_{m}\right\}, \alpha \in(0,1], \\
\Delta u\left(t_{k}\right)=u\left(t_{k}^{+}\right)-u\left(t_{k}^{-}\right)=I_{k}\left(y\left(t_{k}^{-}\right)\right), \quad k=1,2, \ldots, m, \\
u(0)=a .
\end{gathered}
$$

One can notice that the problem (2) contains a multiple base points FDE with base points $0, t_{1}, t_{2}, \ldots, t_{m}$ (impulse points).

In [28], the authors studied the existence and uniqueness of solutions of the following initial value problem of fractional order differential equations:

$$
\begin{gathered}
{ }^{c} D^{\alpha} u(t)=f(t, u(t)), \quad t \in(0, b] \backslash\left\{t_{1}, \ldots, t_{m}\right\}, \alpha \in(1,2], \\
\Delta u\left(t_{k}^{+}\right)=I_{k}\left(u\left(t_{k}^{-}\right)\right), \quad k=1,2, \ldots, m, \\
\Delta u^{\prime}\left(t_{k}\right)=J_{k}\left(u\left(t_{k}^{-}\right)\right), \quad k=1,2, \ldots, m, \\
u(0)=u_{0}, \quad u^{\prime}(0)=u_{1},
\end{gathered}
$$

where the fractional differential equations are a multiple base points FDE with the base points $0, t_{1}, t_{2}, \ldots, t_{m}$ (impulse points).

Fečkan et al. [29] studied the existence of solutions of the following initial value problem of impulsive fractional differential equations:

$$
\begin{gathered}
D_{0^{+}}^{\alpha} u(t)=f(t, u(t)), \quad t \in(0, b] \backslash\left\{t_{1}, \ldots, t_{m}\right\}, \alpha \in(0,1], \\
u\left(t_{k}^{+}\right)=I_{k}\left(y\left(t_{k}^{-}\right)\right), \quad k=1,2, \ldots, m, \\
u(0)=a,
\end{gathered}
$$

where $0<t_{1}<t_{2}<\cdots<t_{m}<b, b>0$ is a fixed real number, $f:[0, b] \times R \rightarrow R$ is jointly continuous, $I_{k}: R \rightarrow R(k=1$, $2, \ldots, m)$ are continuous functions, $u\left(t_{k}^{+}\right)=\lim _{t \rightarrow t_{k}^{+}} u(t)$ and $u\left(t_{k}^{-}\right)=\lim _{t \rightarrow t_{k}^{-}} u(t)$ and $u^{\prime}\left(t_{k}^{+}\right)=\lim _{t \rightarrow t_{k}^{+}} u^{\prime}(t)$. Observe that the fractional differential equation in (4) is a single base point
FDE with the base point $t=0$. So the impulse points are different from the base point.

Liu and Ahmad [30] studied a problem of multi-term and multiorder quasi-Laplacian singular fractional differential equations:

$$
\begin{aligned}
& D_{0^{+}}^{\beta}\left[\Phi\left(\rho(t) D_{0^{+}}^{\alpha} x(t)\right)\right] \\
& \quad+q(t) f\left(t, x(t), D_{0^{+}}^{\alpha} x(t)\right)=0, \quad t \in(0,+\infty), \\
& \lim _{t \rightarrow 0} t^{1-\alpha} x(t)=\int_{0}^{+\infty} m(t) g\left(t, x(t), D_{0^{+}}^{\alpha} x(t)\right) d t, \\
& \quad \lim _{t \rightarrow+\infty} I_{0^{+}}^{1-\beta} \Phi\left(\rho(t) D_{0^{+}}^{\alpha} x(t)\right) \\
& \quad=\int_{0}^{+\infty} n(t) h\left(t, x(t), D_{0^{+}}^{\alpha} x(t)\right) d t, \\
& \Delta x\left(t_{k}\right)=I_{k}\left(t_{k}, x\left(t_{k}\right), D_{0^{+}}^{\alpha} x\left(t_{k}\right)\right), \quad k=1,2, \ldots, \\
& \Delta \Phi\left(\rho\left(t_{k}\right) D_{0^{+}}^{\alpha} x\left(t_{k}\right)\right)=J_{k}\left(t_{k}, x\left(t_{k}\right), D_{0^{+}}^{\alpha} x\left(t_{k}\right)\right), \\
& \quad k=1,2, \ldots,
\end{aligned}
$$

where $1<\alpha, \beta \leq 1,0<t_{1}<t_{2}<\cdots$ are fixed points, $D_{0^{+}}$is the Riemann-Liouville fractional derivative, $\Phi: R \rightarrow$ $R$ is a sup-multiplicative function, $f, g, h$ are impulsive Caratheodory functions, $m, q, n, \rho:(0,1) \rightarrow(0,+\infty)$ are continuous functions, and $I_{k}, J_{k}$ are impulse functions. In (5), the fractional differential equation is a single base point FDE with the base point $t=0$. Clearly the impulse points are different from the base point.

Remark. It is clear from the abovementioned work that IVPs of impulsive fractional differential equations can be categorized into two classes: (a) IVPs of one base point FDEs [20, 29, 30] and (b) IVPs of multiple base points FDEs [12, 27, 28].

In this paper, we study the following two initial value problems (IVPs for short) of nonlinear multi-term FDEs with impulses on half lines:

$$
\begin{gathered}
{ }^{c} D_{0^{+}}^{\alpha} x(t)=q(t) f\left(t, x(t),{ }^{c} D_{0+}^{p} x(t)\right), \quad t \in(0, \infty), \\
x(0)=x_{0}, \\
\Delta x\left(t_{k}\right)=I_{k}\left(t_{k}, x\left(t_{k}\right)\right), \quad k=1,2, \ldots, \\
{ }^{c} D_{*}^{\alpha} x(t)=q(t) f\left(t, x(t),{ }^{c} D_{*}^{p} x(t)\right), \quad t \in(0, \infty), \\
\Delta x\left(t_{k}\right)=I_{k}\left(t_{k}, x\left(t_{k}\right)\right), \quad k=1,2, \ldots,
\end{gathered}
$$

where $x_{0} \in R, \alpha \in(0,1], 0<p<\alpha, 0=t_{0}<t_{1}<t_{2}<$ $t_{3}<\cdots$ with $\lim _{k \rightarrow \infty} t_{k}=\infty,{ }^{c} D_{0^{+}}$is the standard Caputo 
fractional derivative at the base point $t=0, q:(0, \infty) \rightarrow R$ satisfies that there exists $l>-\alpha$ such that $|q(t)| \leq t^{l}$ for all $t \in$ $(0, \infty), q$ may be singular at $t=0,{ }^{c} D_{*}$ is the standard Caputo fractional derivative at the base points $t=t_{k}(k=1,2, \ldots)$; that is, $\left.{ }^{c} D_{*}^{\alpha}\right|_{\left(t_{k}, t_{k+1}\right]} u(t)={ }^{c} D_{t_{k}^{+}}^{\alpha} u(t)$ for all $t \in\left(t_{k}, t_{k+1}\right]$, and $f:[0, \infty) \times R^{2} \rightarrow R$ is a Caratheodory function, $I_{k}:(0, \infty) \times$ $R \rightarrow R(k=1,2, \ldots)$ and $\left\{I_{k}\right\}$ is a Caratheodory function sequence, and $\Delta x\left(t_{k}\right)=\lim _{t \rightarrow t_{k}^{+}} x(t)-\lim _{t \rightarrow t_{k}^{-}} x(t), k=1$, $2, \ldots$..

The salient features of the present work include the following: (i) to establish sufficient conditions for the existence of solutions for the IVP (6) with a single base point and IVP (7) with multiple base points (same as the impulse points). We emphasize that the conditions for the existence of solutions for the IVPs (6) and (7) are different; (ii) the asymptotic behavior of solutions for the problems is studied and the sufficient criterion for every solution to tend to zero as $t \rightarrow \infty$ is established; (iii) the method of proof relies on the Schauder fixed point theorem; (iv) our approach for dealing with impulsive problems at hand is different from the ones employed in earlier work on the topic and thus opens a new avenue for studying impulsive fractional differential equations; (v) as an application, we apply our results to fractional-order logistic models and present sufficient conditions for the existence and asymptotic behavior of solutions of these logistic models.

The paper is organized as follows: the auxiliary material is given in Section 2, the main results are presented in Sections 3 and 4 , while the application of the main results is demonstrated in Section 5.

\section{Preliminaries}

We recall some basic concepts of fractional calculus $[9,10]$ and show auxiliary results.

Define the Gamma function and Beta function, respectively, as

$$
\begin{aligned}
& \Gamma\left(\alpha_{1}\right)=\int_{0}^{+\infty} s^{\alpha_{1}-1} e^{-s} d s, \\
& \mathbf{B}\left(\alpha_{2}, \beta_{2}\right)=\int_{0}^{1}(1-x)^{\alpha_{2}-1} x^{\beta_{2}-1} d x, \\
& \alpha_{1}>0, \quad \alpha_{2}, \beta_{2}>0 .
\end{aligned}
$$

Definition 1 (see [9]). Riemann-Liouville fractional integral of order $\alpha>0$ of a continuous function $f:(0, \infty) \rightarrow R$ is given by

$$
I_{0+}^{\alpha} f(t)=\frac{1}{\Gamma(\alpha)} \int_{0}^{t}(t-s)^{\alpha-1} f(s) d s,
$$

provided that the right-hand side exists.

Definition 2 (see [9]). Caputo's derivative of fractional-order $\alpha$ for a function $f \in A C^{(n-1)}([0, \infty), R)$ is defined by

$$
D_{0^{+}}^{\alpha} f(t)=\frac{1}{\Gamma(n-\alpha)} \int_{0}^{t}(t-s)^{n-\alpha-1} f^{(n)}(s) d s,
$$

for $n-1<\alpha \leq n, n \in N$. If $0<\alpha \leq 1$, then

$$
D_{0^{+}}^{\alpha} f(t)=\frac{1}{\Gamma(1-\alpha)} \int_{0}^{t}(t-s)^{-\alpha} f^{(1)}(s) d s .
$$

Obviously, Caputo's derivative of a constant is zero.

Lemma 3 (see [9]). For $\alpha>0$, the general solution of fractional differential equation ${ }^{c} D_{0^{+}}^{\alpha} x(t)=0$ is given by $x(t)=c_{0}+$ $c_{1} t+c_{2} t^{2}+\cdots+c_{n-1} t^{n-1}$, where $c_{i} \in R, i=0,1,2, \ldots, n-1, n-1<$ $\alpha \leq n$.

Definition 4. A function $x:[0, \infty) \rightarrow R$ is said to be a solution of the IVP (6) if both $\left.x\right|_{\left(t_{k}, t_{k+1}\right]}(k=0,1,2,3, \ldots)$ and $\left.{ }^{c} D_{0^{+}}^{p} x\right|_{\left(t_{k}, t_{k+1}\right]}(k=0,1,2,3, \ldots)$ are continuous, $x$ satisfies the differential equation ${ }^{c} D_{0^{+}}^{\alpha} x(t)=q(t) f\left(t, x(t),{ }^{c} D_{0^{+}}^{p} x(t)\right)$ a.e. on $(0, \infty) \backslash\left\{t_{1}, t_{2}, t_{3}, \ldots\right\}$, and the limits $\lim _{t \rightarrow t_{k}^{+}} x(t)$ and $\lim _{t \rightarrow t_{k}^{+}}{ }^{c} D_{0^{+}}^{p} x(t)(k=0,1,2,3, \ldots)$ exist and the following conditions are satisfied:

$$
\Delta x\left(t_{k}\right)=I_{k}\left(t_{k}, x\left(t_{k}\right)\right), \quad k=1,2, \ldots, x(0)=x_{0} .
$$

Definition 5. A function $x:[0, \infty) \rightarrow R$ is said to be a solution of the IVP (7) if both $\left.x\right|_{\left(t_{k}, t_{k+1}\right]}(k=0,1,2,3, \ldots)$ and $\left.{ }^{c} D_{0^{+}}^{p} x\right|_{\left(t_{k}, t_{k+1}\right]}(k=0,1,2,3, \ldots)$ are continuous, $x$ satisfies the differential equation ${ }^{c} D_{t_{k}^{+}}^{\alpha} x(t)=q(t) f(t, x(t)$,

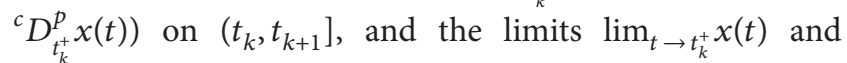
$\lim _{t \rightarrow t_{k}^{+}}{ }^{c} D_{0^{+}}^{p} x(t)(k=0,1,2,3, \ldots)$ exist and the following conditions are satisfied:

$$
\Delta x\left(t_{k}\right)=I_{k}\left(t_{k}, x\left(t_{k}\right)\right), \quad k=1,2, \ldots, x(0)=x_{0} .
$$

Choose $\sigma>\max \{0, \alpha+l\}$ and $\mu>\max \{\sigma, \sigma-\alpha-l\}$. Let

$$
X=\left\{\begin{array}{cc}
\left.x\right|_{\left(t_{k}, t_{k+1}\right]} \in C^{0}\left(t_{k}, t_{k+1}\right], \quad k=0,1,2, \ldots, \\
& \left.{ }^{c} D_{0^{+}}^{p} x\right|_{\left(t_{k}, t_{k+1}\right]} \in C^{0}\left(t_{k}, t_{k+1}\right], \quad k=0,1,2, \ldots, \\
& \frac{t^{\sigma-\alpha-l}}{(1+t)(1+t \mu)} x(t) \text { is bounded on }(0, \infty) \\
& \frac{t^{p+\sigma-\alpha-l}}{1+t \mu}{ }^{c} D_{0^{+}}^{p} x(t) \text { is bounded on }(0, \infty) .
\end{array}\right\} .
$$


For $x \in X$, define the norm on $X$ as

$$
\begin{array}{r}
\|x\|=\max \left\{\sup _{t \in(0, \infty)} \frac{t^{\sigma-\alpha-l}}{(1+t)\left(1+t^{\mu}\right)}|x(t)|,\right. \\
\left.\sup _{t \in(0, \infty)} \frac{t^{p+\sigma-\alpha-l}}{1+t^{\mu}}\left|{ }^{c} D_{0^{+}}^{p} x(t)\right|\right\} .
\end{array}
$$

It is easy to show that $X$ is a real Banach space.

Definition 6. $f:[0,+\infty) \times R^{2} \rightarrow R$ is called a Caratheodory function if it satisfies the following assumptions:

(i) $(t, x, y) \rightarrow f\left(t,\left((1+t)\left(1+t^{\mu}\right) / t^{\sigma-\alpha-l}\right) x,((1+\right.$ $\left.\left.\left.t^{\mu}\right) / t^{p+\sigma-\alpha-l}\right) y\right)$ is continuous on $[0,+\infty) \times R^{2}$;

(ii) for each $r>0$, there exists a constant $M_{r}>0$ such that $|x|,|y| \leq r$ implies that

$$
\left|f\left(t, \frac{(1+t)\left(1+t^{\mu}\right)}{t^{\sigma-\alpha-l}} x, \frac{1+t^{\mu}}{t^{p+\sigma-\alpha-l}} y\right)\right| \leq M_{r}, \quad t \in[0, \infty) .
$$

Definition 7. $\left\{I_{k}\right\}$ is called a Caratheodory function sequence if it satisfies the following assumptions:

(i) $x \rightarrow I_{k}\left(t_{k},\left(\left(1+t_{k}\right)\left(1+t_{k}^{\mu}\right) / t_{k}^{\sigma-\alpha-l}\right) x\right)$ is continuous on $R$ for each $k=1,2,3, \ldots$;

(ii) for each $r>0$, there exist constants $M_{r k}>0$ such that $|x| \leq r$ implies that

$$
\left|I_{k}\left(t_{k}, \frac{\left(1+t_{k}\right)\left(1+t_{k}^{\mu}\right)}{t_{k}^{\sigma-\alpha-l}} x\right)\right| \leq M_{r k}, \quad \sum_{k=1}^{\infty} M_{r k}<\infty .
$$

If $b>a>0$, then we have

$$
\sup _{t \in(0, \infty)} \frac{t^{a}}{1+t^{b}}=\frac{1}{b} a^{a / b}(b-a)^{(b-a) / b}=: M_{a, b} \text {. }
$$

Lemma 8. Suppose that $f$ is a Caratheodory function and $\left\{I_{k}\right\}$ is a Caratheodory function sequence on $X$. Then $x \in X$ is a solution of

$$
\begin{gathered}
{ }^{c} D_{0^{+}}^{\alpha} x(t)=q(t) f\left(t, x(t),{ }^{c} D_{0^{+}}^{p} x(t)\right), \quad t \in(0, \infty), \\
x(0)=x_{0}, \\
\Delta x\left(t_{k}\right)=I_{k}\left(t_{k}, x\left(t_{k}\right)\right), \quad k=1,2, \ldots,
\end{gathered}
$$

if and only if $x \in X$ is a solution of the fractional integral equation

$$
\begin{aligned}
x(t)=x_{0}+\int_{0}^{t} \frac{(t-s)^{\alpha-1}}{\Gamma(\alpha)} q(s) & \\
& \times f\left(s, x(s),{ }^{c} D_{0^{+}}^{p} x(s)\right) d s \\
& +\sum_{j=1}^{k} I_{j}\left(t_{j}, x\left(t_{j}\right)\right), \quad t \in\left(t_{k}, t_{k+1}\right], k=0,1,2, \ldots
\end{aligned}
$$

Proof. For $x \in X$ and $r>0$, we have

$$
\begin{aligned}
\max \left\{\sup _{t \in(0, \infty)} \frac{t^{\sigma-\alpha-l}}{(1+t)\left(1+t^{\mu}\right)}|x(t)|,\right. \\
\left.\sup _{t \in(0, \infty)} \frac{t^{p+\sigma-\alpha-l}}{1+t^{\mu}}\left|{ }^{c} D_{0^{+}}^{p} x(t)\right|\right\}=r .
\end{aligned}
$$

Since $f$ is a Caratheodory function and $\left\{I_{k}\right\}$ is a Caratheodory function sequence, therefore, there exist $M_{r}>0$ and $M_{r k}>0$ such that

$$
\begin{aligned}
& \left|f\left(t, x(t),{ }^{c} D_{0^{+}}^{p} x(t)\right)\right| \\
& =\mid f\left(t, \frac{(1+t)\left(1+t^{\mu}\right)}{t^{\sigma-\alpha-l}} \frac{t^{\sigma-\alpha-l}}{(1+t)\left(1+t^{\mu}\right)} x(t),\right. \\
& \quad \leq M_{r}, \quad t \in[0, \infty), \\
& \left.\left|I_{k}\left(t_{k}, x\left(t_{k}\right)\right)\right| \quad \mid t^{\mu} t^{p+\sigma-\alpha-\alpha} \frac{t^{c}-l}{1+t^{\mu}} D_{0^{+}}^{p} x(t)\right) \mid \\
& \quad \mid t_{k}, \frac{\left(1+t_{k}\right)\left(1+t_{k}^{\mu}\right)}{t_{k}^{\sigma-\alpha-l}} \\
& \left.\leq M_{r k}, \quad k \frac{t_{k}^{\sigma-\alpha-l}}{\left(1+t_{k}\right)\left(1+t_{k}^{\mu}\right)} x\left(t_{k}\right)\right) \mid \\
& k=1,2,3, \ldots, \sum_{k=1}^{\infty} M_{r k}<\infty .
\end{aligned}
$$

Let us assume that $x$ satisfies (48). Then, by Lemma 3, the solution of (48) can be written as

$$
\begin{array}{r}
x(t)=\int_{0}^{t} \frac{(t-s)^{\alpha-1}}{\Gamma(\alpha)} q(s) f\left(s, x(s),{ }^{c} D_{0^{+}}^{p} x(s)\right) d s+c_{k}, \\
t \in\left(t_{k}, t_{k+1}\right], \quad k=0,1,2, \ldots
\end{array}
$$

Observe that

$$
\begin{aligned}
& \left|\int_{0}^{t} \frac{(t-s)^{\alpha-1}}{\Gamma(\alpha)} q(s) f\left(s, x(s),{ }^{c} D_{0^{+}}^{p} x(s)\right) d s\right| \\
& \quad \leq M_{r} \int_{0}^{t} \frac{(t-s)^{\alpha-1}}{\Gamma(\alpha)} s^{l} d s \\
& \quad=M_{r} t^{\alpha+l} \int_{0}^{1} \frac{(1-w)^{\alpha-1}}{\Gamma(\alpha)} d w \longrightarrow 0 \text { as } t \longrightarrow 0 .
\end{aligned}
$$


From $x(0)=x_{0}$ and $\Delta y\left(t_{k}\right)=I_{k}\left(t_{k}, x\left(t_{k}\right)\right)$, we get $c_{0}=x_{0}$ and

$$
\begin{gathered}
\int_{0}^{t_{k}} \frac{\left(t_{k}-s\right)^{\alpha-1}}{\Gamma(\alpha)} q(s) f\left(s, x(s),{ }^{c} D_{0^{+}}^{p} x(s)\right) d s+c_{k} \\
-\left(\int_{0}^{t_{k}} \frac{\left(t_{k}-s\right)^{\alpha-1}}{\Gamma(\alpha)} q(s)\right. \\
\left.\quad \times f\left(s, x(s),{ }^{c} D_{0^{+}}^{p} x(s)\right) d s+c_{k-1}\right) \\
=I_{k}\left(t_{k}, x\left(t_{k}\right)\right) .
\end{gathered}
$$

This implies that

$$
\begin{aligned}
c_{k} & =c_{k-1}+I_{k}\left(t_{k}, x\left(t_{k}\right)\right) \\
& =x_{0}+\sum_{j=1}^{k} I_{j}\left(t_{j}, x\left(t_{j}\right)\right), \quad k=0,1,2, \ldots
\end{aligned}
$$

Thus, we have

$$
\begin{aligned}
x(t)= & \int_{0}^{t} \frac{(t-s)^{\alpha-1}}{\Gamma(\alpha)} q(s) f\left(s, x(s),{ }^{c} D_{0^{+}}^{p} x(s)\right) d s \\
& +x_{0}+\sum_{j=1}^{k} I_{j}\left(t_{j}, x\left(t_{j}\right)\right) .
\end{aligned}
$$

Hence, $x$ satisfies (49). Next, we show that $x \in X$. Indeed

$$
{ }^{c} D_{0^{+}}^{p} x(t)=\int_{0}^{t} \frac{(t-s)^{\alpha-p-1}}{\Gamma(\alpha-p)} q(s) f\left(s, x(s),{ }^{c} D_{0^{+}}^{p} x(s)\right) d s .
$$

It is easy to see that

$$
\begin{array}{r}
\left.x\right|_{\left(t_{k}, t_{k+1}\right]} \in C^{0}\left(t_{k}, t_{k+1}\right], \\
\left.{ }^{c} D_{0^{+}}^{p} x\right|_{\left(t_{k}, t_{k+1}\right]} \\
\in C^{0}\left(t_{k}, t_{k+1}\right], \\
k=0,1,2, \ldots .
\end{array}
$$

Furthermore, for $t \in\left(t_{k}, t_{k+1}\right]$, we have

$$
\begin{aligned}
& \frac{t^{\sigma-\alpha-l}}{(1+t)\left(1+t^{\mu}\right)}|x(t)| \\
& \leq \frac{t^{\sigma-\alpha-l}}{1+t^{\mu}} \mid \int_{0}^{t} \frac{(t-s)^{\alpha-1}}{\Gamma(\alpha)} q(s) f\left(s, x(s),{ }^{c} D_{0^{+}}^{p} x(s)\right) d s \\
& +x_{0}+\sum_{j=1}^{k} I_{j}\left(t_{j}, x\left(t_{j}\right)\right) \mid
\end{aligned}
$$

$$
\begin{aligned}
& \leq \frac{t^{\sigma-\alpha-l}}{(1+t)\left(1+t^{\mu}\right)} \\
& \times \int_{0}^{t} \frac{(t-s)^{\alpha-1}}{\Gamma(\alpha)}\left|q(s) f\left(s, x(s),{ }^{c} D_{0^{+}}^{p} x(s)\right)\right| d s \\
& +\frac{t^{\sigma-\alpha-l}}{(1+t)\left(1+t^{\mu}\right)}\left|x_{0}\right| \\
& +\frac{t^{\sigma-\alpha-l}}{(1+t)\left(1+t^{\mu}\right)} \sum_{j=1}^{k}\left|I_{j}\left(t_{j}, x\left(t_{j}\right)\right)\right| \\
& \leq \frac{t^{\sigma-\alpha-l}}{(1+t)\left(1+t^{\mu}\right)} \int_{0}^{t} \frac{(t-s)^{\alpha-1}}{\Gamma(\alpha)} M_{r} s^{l} d s \\
& +M_{\sigma-\alpha-l, \mu}\left|x_{0}\right|+M_{\sigma-\alpha-l, \mu} \sum_{j=1}^{k} M_{r k} \\
& =\frac{M_{r} t^{\sigma}}{1+t} \int_{0}^{1} \frac{(1-w)^{\alpha-1}}{\Gamma(\alpha)} w^{l} d w+\left|x_{0}\right|+\sum_{j=1}^{k} M_{r k} \\
& \leq M_{r} M_{\sigma, \mu} \frac{\mathbf{B}(\alpha, l+1)}{\Gamma(\alpha)}+M_{\sigma-\alpha-l, \mu}\left|x_{0}\right| \\
& +M_{\sigma-\alpha-l, \mu} \sum_{j=1}^{\infty} M_{r k}<\infty, \\
& \frac{t^{\sigma+p-\alpha-l}}{1+t^{\mu}}\left|D_{0^{+}}^{p} x(t)\right| \\
& =\frac{t^{\sigma+p-\alpha-l}}{1+t^{\mu}} \\
& \times\left|\int_{0}^{t} \frac{(t-s)^{\alpha-p-1}}{\Gamma(\alpha-p)} q(s) f\left(s, x(s),{ }^{c} D_{0^{+}}^{p} x(s)\right) d s\right|
\end{aligned}
$$

$$
\begin{aligned}
& \leq \frac{t^{\sigma+p-\alpha-l}}{1+t^{\mu}} \int_{0}^{t} \frac{(t-s)^{\alpha-p-1}}{\Gamma(\alpha-p)} M_{r} s^{l} d s \\
& =\frac{M_{r} t^{\sigma}}{1+t^{\mu}} \int_{0}^{1} \frac{(1-w)^{\alpha-p-1}}{\Gamma(\alpha-p)} w^{l} d w \\
& \leq M_{r} M_{\sigma, \mu} \frac{\mathbf{B}(\alpha-p, l+1)}{\Gamma(\alpha-p)}<\infty .
\end{aligned}
$$

This implies that $x \in X$. Conversely, suppose that $x$ satisfies (49). By a direct computation, it follows that the solution given by (49) satisfies the problem (48). This completes the proof.

Choose $\sigma>\max \{0, \alpha+l\}$ and $\mu>\max \{\sigma, \sigma-\alpha-l\}$ and define 


$$
Y=\left\{\begin{array}{c}
\left.x\right|_{\left(t_{k}, t_{k+1}\right]} \in C^{0}\left(t_{k}, t_{k+1}\right], \quad k=0,1,2, \ldots, \\
\\
\left.{ }^{c} D_{t_{k}^{+}}^{p} x\right|_{\left(t_{k}, t_{k+1}\right]} \in C^{0}\left(t_{k}, t_{k+1}\right], \quad k=0,1,2, \ldots, \\
\\
\left\{\sup _{t \in(t k, t k+1]} \frac{t^{\sigma-\alpha-l}}{(1+t)(1+t \mu)} x(t) \text { is bounded on }(0, \infty)\right. \\
\end{array}\right\} .
$$

For $x \in Y$, we define the norm on $Y$ as

$$
\begin{aligned}
&\|x\|=\max \left\{\sup _{t \in(0, \infty)} \frac{t^{\sigma-\alpha-l}}{(1+t)\left(1+t^{\mu}\right)}|x(t)|,\right. \\
&\left.\sup _{k=0,1,2, \ldots}\left\{\sup _{t \in\left(t_{k}, t_{k+1}\right.} \frac{t^{p+\sigma-\alpha-l}}{1+t^{\mu}}\left|D^{c} D_{t^{+}}^{p} x(t)\right|\right\}\right\} .
\end{aligned}
$$

It is easy to show that $Y$ is a real Banach space.

Lemma 9. Suppose that $f$ is a Caratheodory function and $\left\{I_{k}\right\}$ is a Caratheodory function sequence, $x \in Y$ and $\lambda_{0}=$ : $\inf _{k=0,1,2, \ldots}\left(t_{k}-t_{k-1}\right)>0$. Then $x \in Y$ is a solution of the problem

$$
\begin{gathered}
{ }^{c} D_{*}^{\alpha} y(t)=q(t) f\left(t, x(t),{ }^{c} D_{*}^{p} x(t)\right), \quad t \in(0, \infty), \\
y(0)=x_{0}, \\
\Delta y\left(t_{k}\right)=I_{k}\left(t_{k}, x\left(t_{k}\right)\right), \quad k=1,2, \ldots,
\end{gathered}
$$

if and only if $x \in Y$ is a solution of the fractional integral equation

$$
\begin{aligned}
x(t)= & \int_{t_{k}}^{t} \frac{(t-s)^{\alpha-1}}{\Gamma(\alpha)} q(s) f\left(s, x(s),{ }^{c} D_{t_{k}^{+}}^{p} x(s)\right) d s \\
& +x_{0}+\sum_{j=1}^{k} I_{j}\left(t_{j}, x\left(t_{j}\right)\right) \\
& +\sum_{j=1}^{k} \int_{t_{j-1}}^{t_{j}} \frac{\left(t_{j}-s\right)^{\alpha-1}}{\Gamma(\alpha)} q(s) f\left(s, x(s),{ }^{c} D_{t_{j-1}^{+}}^{p} x(s)\right) d s, \\
& \quad t \in\left(t_{k}, t_{k+1}\right], \quad k=0,1,2, \ldots
\end{aligned}
$$

Proof. For $x \in Y$, we have that there exists $r>0$ such that

$$
\begin{aligned}
\max & \left\{\sup _{t \in(0, \infty)} \frac{t^{\sigma-\alpha-l}}{(1+t)\left(1+t^{\mu}\right)}|x(t)|,\right. \\
& \left.\sup _{n=0,1,2, \ldots . . t \in\left(t_{k}, t_{k+1}\right]} \frac{t^{p+\sigma-\alpha-l}}{(1+t)\left(1+t^{\mu}\right)}\left|{ }^{c} D_{t_{k}^{+}}^{p} x(t)\right|\right\}=r .
\end{aligned}
$$

Since $f$ is a Caratheodory function and $\left\{I_{k}\right\}$ is a Caratheodory function sequence, then there exist $M_{r}>0$ and $M_{r k}>0$ such that

$$
\begin{aligned}
& \left|f\left(t, x(t),{ }^{c} D_{t_{k}^{+}}^{p} x(t)\right)\right| \\
& =\mid f\left(t, \frac{(1+t)\left(1+t^{\mu}\right)}{t^{\sigma-\alpha-l}} \frac{t^{\sigma-\alpha-l}}{(1+t)\left(1+t^{\mu}\right)} x(t),\right. \\
& \left.\quad \frac{1+t^{\mu}}{t^{p+\sigma-\alpha-l}} \frac{t^{p+\sigma-\alpha-l}}{1+t^{\mu}}{ }^{c} D_{t^{+}}^{p} x(t)\right) \mid \\
& \quad \leq M_{r}, \quad t \in[0, \infty), \\
& \quad=\left|I_{k}\left(t_{k}, x\left(t_{k}\right)\right)\right| \frac{\left(1+t_{k}\right)\left(1+t_{k}^{\mu}\right)}{t_{k}^{\sigma-\alpha-l}} \\
& \left.\quad \times \frac{t_{k}^{\sigma-\alpha-l}}{\left(1+t_{k}\right)\left(1+t_{k}^{\mu}\right)} x\left(t_{k}\right)\right) \mid \\
& \leq M_{r k}, \quad k=1,2,3, \ldots, \sum_{k=1}^{\infty} M_{r k}<\infty .
\end{aligned}
$$

Assume that $x$ satisfies the problem (50). Then, in view of Lemma 3, we can write the solution of (50) as

$$
\begin{array}{r}
x(t)=\int_{t_{k}}^{t} \frac{(t-s)^{\alpha-1}}{\Gamma(\alpha)} q(s) f\left(s, x(s),{ }^{c} D_{t_{k}^{+}}^{p} x(s)\right) d s+c_{k}, \\
t \in\left(t_{k}, t_{k+1}\right], \quad k=0,1,2, \ldots
\end{array}
$$


From $x(0)=x_{0}$, we get $c_{0}=x_{0}$. Since

$$
\begin{gathered}
\left|\int_{t_{k}}^{t} \frac{(t-s)^{\alpha-1}}{\Gamma(\alpha)} q(s) f\left(s, x(s),{ }^{c} D_{t_{k}^{+}}^{p} x(s)\right) d s\right| \\
\leq M_{r} \int_{t_{k}}^{t} \frac{(t-s)^{\alpha-1}}{\Gamma(\alpha)} s^{l} d s \\
\quad=M_{r} t^{\alpha+l} \int_{t_{k} / t}^{1} \frac{(1-w)^{\alpha-1}}{\Gamma(\alpha)} w^{l} d w \longrightarrow 0 \\
\text { as } t \longrightarrow t_{k}^{+}, \quad k=1,2,3, \ldots
\end{gathered}
$$

and $\Delta y\left(t_{k}\right)=I_{k}\left(t_{k}, x\left(t_{k}\right)\right)$, we get

$$
\begin{gathered}
c_{k}-\left(\int_{t_{k-1}}^{t_{k}} \frac{\left(t_{k}-s\right)^{\alpha-1}}{\Gamma(\alpha)} q(s) f\left(s, x(s),{ }^{c} D_{t_{k-1}^{+}}^{p} x(s)\right) d s\right. \\
\left.+c_{k-1}\right)=I_{k}\left(t_{k}, x\left(t_{k}\right)\right),
\end{gathered}
$$

which gives

$$
\begin{aligned}
& c_{k}= c_{k-1}+I_{k}\left(t_{k}, x\left(t_{k}\right)\right) \\
&+\int_{t_{k-1}}^{t_{k}} \frac{\left(t_{k}-s\right)^{\alpha-1}}{\Gamma(\alpha)} q(s) f\left(s, x(s),{ }^{c} D_{t_{k-1}^{+}}^{p} x(s)\right) d s \\
&= x_{0}+\sum_{j=1}^{k} I_{j}\left(t_{j}, x\left(t_{j}\right)\right) \\
&+\sum_{j=1}^{k} \int_{t_{j-1}}^{t_{j}} \frac{\left(t_{j}-s\right)^{\alpha-1}}{\Gamma(\alpha)} q(s) f\left(s, x(s),{ }^{c} D_{t_{j-1}^{+}}^{p} x(s)\right) d s, \\
& k=0,1,2, \ldots
\end{aligned}
$$

Hence the solution of the problem (50) is

$$
\begin{aligned}
x(t)= & \int_{t_{k}}^{t} \frac{(t-s)^{\alpha-1}}{\Gamma(\alpha)} q(s) f\left(s, x(s),{ }^{c} D_{t_{k}^{+}}^{p} x(s)\right) d s \\
& +x_{0}+\sum_{j=1}^{k} I_{j}\left(t_{j}, x\left(t_{j}\right)\right) \\
& +\sum_{j=1}^{k} \int_{t_{j-1}}^{t_{j}} \frac{\left(t_{j}-s\right)^{\alpha-1}}{\Gamma(\alpha)} q(s) f\left(s, x(s),{ }^{c} D_{t_{j-1}^{+}}^{p} x(s)\right) d s, \\
& \quad t \in\left(t_{k}, t_{k+1}\right], \quad k=0,1,2, \ldots
\end{aligned}
$$

Next, we need to show that $x \in Y$. Clearly,

$$
\begin{gathered}
{ }^{c} D_{t_{k}^{+}}^{p} x(t)=\int_{0}^{t} \frac{(t-s)^{\alpha-p-1}}{\Gamma(\alpha-p)} q(s) f\left(s, x(s),{ }^{c} D_{t_{k}^{+}}^{p} x(s)\right) d s \\
\left.x\right|_{\left(t_{k}, t_{k+1}\right]} \in C^{0}\left(t_{k}, t_{k+1}\right], \\
\left.{ }^{c} D_{t_{k}^{+}}^{p} x\right|_{\left(t_{k}, t_{k+1}\right]} \in C^{0}\left(t_{k}, t_{k+1}\right] \\
k=0,1,2, \ldots
\end{gathered}
$$

Furthermore, for $t \in\left(t_{k}, t_{k+1}\right]$, we have

$$
\begin{aligned}
& \frac{t^{\sigma-\alpha-l}}{(1+t)\left(1+t^{\mu}\right)}|x(t)| \\
& =\frac{t^{\sigma-\alpha-l}}{(1+t)\left(1+t^{\mu}\right)} \\
& \times \mid \int_{t_{k}}^{t} \frac{(t-s)^{\alpha-1}}{\Gamma(\alpha)} q(s) f\left(s, x(s),{ }^{c} D_{t_{k}^{+}}^{p} x(s)\right) d s \\
& +x_{0}+\sum_{j=1}^{k} I_{j}\left(t_{j}, x\left(t_{j}\right)\right) \\
& +\sum_{j=1}^{k} \int_{t_{j-1}}^{t_{j}} \frac{\left(t_{j}-s\right)^{\alpha-1}}{\Gamma(\alpha)} q(s) \\
& \times f\left(s, x(s),{ }^{c} D_{t_{j-1}^{+}}^{p} x(s)\right) d s \\
& \leq \frac{t^{\sigma-\alpha-l}}{(1+t)\left(1+t^{\mu}\right)} \int_{t_{k}}^{t} \frac{(t-s)^{\alpha-1}}{\Gamma(\alpha)} M_{r} s^{l} d s \\
& +M_{\sigma-\alpha-l, \mu}\left|x_{0}\right|+M_{\sigma-\alpha-l, \mu} \sum_{j=1}^{k} M_{r k} \\
& +\frac{t^{\sigma-\alpha-l}}{(1+t)\left(1+t^{\mu}\right)} \sum_{j=1}^{k} \int_{t_{j-1}}^{t_{j}} \frac{\left(t_{j}-s\right)^{\alpha-1}}{\Gamma(\alpha)} M_{r} s^{l} d s \\
& \leq M_{r} \frac{t^{\sigma}}{(1+t)\left(1+t^{\mu}\right)} \int_{t_{k} / t}^{1} \frac{(1-w)^{\alpha-1}}{\Gamma(\alpha)} w^{l} d w \\
& +M_{\sigma-\alpha-l, \mu}\left|x_{0}\right|+M_{\sigma-\alpha-l, \mu} \sum_{j=1}^{\infty} M_{r k} \\
& +\frac{t^{\sigma-\alpha-l}}{(1+t)\left(1+t^{\mu}\right)} M_{r} \\
& \times \sum_{j=1}^{k} t_{j}^{\alpha+l} \int_{t_{j-1} / t_{j}}^{1} \frac{(1-w)^{\alpha-1}}{\Gamma(\alpha)} w^{l} d w \\
& \leq M_{r} M_{\sigma, \mu} \int_{0}^{1} \frac{(1-w)^{\alpha-1}}{\Gamma(\alpha)} w^{l} d w
\end{aligned}
$$




$$
\begin{aligned}
& +M_{\sigma-\alpha-l, \mu}\left|x_{0}\right|+M_{\sigma-\alpha-l, \mu} \sum_{j=1}^{\infty} M_{r k} \\
& +M_{r} \sum_{j=1}^{k} \frac{t^{\sigma-\alpha-l} t_{j}^{\alpha+l}}{(1+t)\left(1+t^{\mu}\right)} \\
& \times \int_{t_{j-1} / t_{j}}^{1} \frac{(1-w)^{\alpha-1}}{\Gamma(\alpha)} w^{l} d w \\
& \leq M_{r} M_{\sigma, \mu} \frac{\mathbf{B}(\alpha, l+1)}{\Gamma(\alpha)}+M_{\sigma-\alpha-l, \mu}\left|x_{0}\right| \\
& +M_{\sigma-\alpha-l, \mu} \sum_{j=1}^{\infty} M_{r k}+M_{r} \sum_{j=1}^{k} \frac{t^{\sigma-\alpha-l} t_{j}^{\alpha+l}}{t^{\mu+1}} \\
& \times \int_{0}^{1} \frac{(1-w)^{\alpha-1}}{\Gamma(\alpha)} w^{l} d w \\
& \leq M_{r} M_{\sigma, \mu} \frac{\mathbf{B}(\alpha, l+1)}{\Gamma(\alpha)}+M_{\sigma-\alpha-l, \mu}\left|x_{0}\right| \\
& +M_{\sigma-\alpha-l, \mu} \sum_{j=1}^{\infty} M_{r k} \\
& +M_{r} \sum_{j=1}^{k} \frac{t_{j}^{\alpha+l}}{t_{j}^{\mu+1-\sigma+\alpha+l}} \frac{\mathbf{B}(\alpha, l+1)}{\Gamma(\alpha)} \\
& \leq M_{r} M_{\sigma, \mu} \frac{\mathbf{B}(\alpha, l+1)}{\Gamma(\alpha)}+M_{\sigma-\alpha-l, \mu}\left|x_{0}\right| \\
& +M_{\sigma-\alpha-l, \mu} \sum_{j=1}^{\infty} M_{r k} \\
& +M_{r} \sum_{j=1}^{k} \frac{1}{t_{j}^{\mu+1-\sigma}} \frac{\mathbf{B}(\alpha, l+1)}{\Gamma(\alpha)} .
\end{aligned}
$$

Since $t_{j}-t_{j-1} \geq \lambda_{0}$ and $t_{0}=0$, we get $t_{j} \geq j \lambda_{0}$ for all $j=0$, $1,2, \ldots$. Then

$$
\begin{aligned}
& \frac{t^{\sigma-\alpha-l}}{(1+t)\left(1+t^{\mu}\right)}|x(t)| \\
& \leq M_{r} M_{\sigma, \mu} \frac{\mathbf{B}(\alpha, l+1)}{\Gamma(\alpha)}+M_{\sigma-\alpha-l, \mu}\left|x_{0}\right| \\
& \quad+M_{\sigma-\alpha-l, \mu} \sum_{j=1}^{\infty} M_{r k} \\
& +M_{r} \frac{1}{\lambda_{0}^{\mu+1-\sigma}} \frac{\mathbf{B}(\alpha, l+1)}{\Gamma(\alpha)} \sum_{j=1}^{\infty} \frac{1}{j^{\mu+1-\sigma}}, \\
& \quad t \in\left(t_{k}, t_{k+1}\right], \quad k=0,1,2, \ldots
\end{aligned}
$$

So

$$
\frac{t^{\sigma-\alpha-l}}{(1+t)\left(1+t^{\mu}\right)}|x(t)| \text { is bounded on }(0, \infty) \text {. }
$$

Moreover, for $t \in\left(t_{k}, t_{k+1}\right]$, we get

$$
\begin{aligned}
& \frac{t^{p+\sigma-\alpha-l}}{1+t^{\mu}}\left|D_{t_{k}^{+}}^{p} x(t)\right| \\
& =\frac{t^{p+\sigma-\alpha-l}}{1+t^{\mu}} \mid \int_{t_{k}}^{t} \frac{(t-s)^{\alpha-p-1}}{\Gamma(\alpha-p)} q(s) \\
& \quad \times f\left(s, x(s),{ }^{c} D_{t_{k}^{+}}^{p} x(s)\right) d s \mid \\
& \leq \frac{t^{p+\sigma-\alpha-l}}{1+t^{\mu}} \int_{t_{k}}^{t} \frac{(t-s)^{\alpha-p-1}}{\Gamma(\alpha-p)} M_{r} s^{l} d s \\
& =\frac{M_{r} t^{\sigma}}{1+t^{\mu}} \int_{t_{k} / t}^{1} \frac{(1-w)^{\alpha-p-1}}{\Gamma(\alpha-p)} w^{l} d w \\
& \leq M_{r} M_{\sigma, \mu} \frac{\mathbf{B}(\alpha-p, l+1)}{\Gamma(\alpha-p)}<\infty, \\
& t \in\left(t_{k}, t_{k+1}\right], \quad k=0,1,2, \ldots
\end{aligned}
$$

So

$$
\frac{t^{p+\sigma-\alpha-l}}{1+t^{\mu}}\left|{ }^{c} D_{t_{k}^{+}}^{p} y(t)\right| \text { is bounded on }(0, \infty) .
$$

Thus, $x \in Y$. Conversely, assume that $x$ satisfies (51). Then, by direct computation, it follows that the solution given by (51) satisfies the problem (50). This completes the proof.

\section{Existence Results for an IVP with a Single Base Point}

In this section, we discuss the existence and uniqueness of solutions for the single base point IVP (6). The asymptotic behaviour of solutions of IVP (6) is also investigated.

In relation to the IVP (6), we define an operator $T: X \rightarrow$ $X$ by

$$
\begin{aligned}
(T x)(t)=\int_{0}^{t} & \frac{(t-s)^{\alpha-1}}{\Gamma(\alpha)} q(s) \\
& \times f\left(s, x(s),{ }^{c} D_{0^{+}}^{p} x(s)\right) d s+x_{0} \\
& +\sum_{j=1}^{k} I_{j}\left(t_{j}, x\left(t_{j}\right)\right), \\
& \quad t \in\left(t_{k}, t_{k+1}\right], \quad k=0,1,2, \ldots
\end{aligned}
$$

Lemma 10. Let $f$ be a Caratheodory function and let $\left\{I_{k}\right\}$ be a Caratheodory function sequence. Then (i) $T: X \rightarrow X$ is well defined; (ii) the fixed point of the operator $T$ coincides with the solution of IVP (6); (iii) $T: X \rightarrow X$ is completely continuous. 
Proof. (i) For $x \in X$, let

$$
\begin{aligned}
r=\max \left\{\sup _{t \in(0, \infty)} \frac{t^{\sigma-\alpha-l}}{(1+t)\left(1+t^{\mu}\right)}|x(t)|,\right. \\
\left.\sup _{t \in(0, \infty)} \frac{t^{p+\sigma-\alpha-l}}{1+t^{\mu}}\left|{ }^{c} D_{0^{+}}^{p} x(t)\right|\right\}<+\infty .
\end{aligned}
$$

Since $f$ is a Caratheodory function, $\left\{I_{k}\right\}$ is Caratheodory function sequence; there exist positive numbers $M_{r}>0$ and $M_{r k}>0(k=1,2, \ldots)$ such that

$$
\begin{gathered}
\left|f\left(t, x(t),{ }^{c} D_{0^{+}}^{p} x(t)\right)\right| \leq M_{r}, \quad t \in[0, \infty), \\
\left|I_{k}\left(t_{k}, x\left(t_{k}\right)\right)\right| \leq M_{r k}, \quad k=1,2, \ldots, \sum_{k=1}^{\infty} M_{r k}<\infty .
\end{gathered}
$$

It is easy to show that

$$
\begin{array}{r}
T x \in C^{0}\left(t_{k}, t_{k+1}\right], \quad{ }^{c} D_{0^{+}}^{p} T x \in C^{0}\left(t_{k}, t_{k+1}\right], \\
k=0,1,2, \ldots .
\end{array}
$$

As in the proof of Lemma 8, it can be shown that both $\left(t^{\sigma-\alpha-l} /(1+t)\left(1+t^{\mu}\right)\right)(T x)(t)$ and $\left(t^{p+\sigma-\alpha-l} /(1+\right.$ $\left.\left.t^{\mu}\right)\right)^{c} D_{0^{+}}^{p}(T x)(t)$ are bounded on $(0, \infty)$.

Hence, $T x \in X$ and consequently $T: X \rightarrow X$ is well defined.

(ii) It follows from Lemma 8 that the fixed point of the operator $T$ coincides with the solution of IVP (6).

(iii) To establish that $T$ is completely continuous, we show that (a) $T$ is continuous, (b) $T$ maps bounded sets of $X$ to bounded sets, and (c) $T$ maps bounded sets of $X$ to relatively compact sets.

(a) In order to show that the operator $T$ is continuous, let $x_{n} \in X$ with $x_{n} \rightarrow x_{0}$ as $n \rightarrow \infty$. We will prove that $T x_{n} \rightarrow T x_{0}$ as $n \rightarrow \infty$. It is easy to see that there exists $r>0$ such that

$$
\begin{gathered}
\max \left\{\sup _{t \in(0, \infty)} \frac{t^{\sigma-\alpha-l}}{(1+t)\left(1+t^{\mu}\right)}\left|x_{n}(t)\right|,\right. \\
\left.\sup _{t \in(0, \infty)} \frac{t^{p+\sigma-\alpha-l}}{1+t^{\mu}}\left|{ }^{c} D_{0^{+}}^{p} x_{n}(t)\right|\right\} \leq r<\infty, \\
n=0,1,2, \ldots, \\
\sup _{t \in(0, \infty)} \frac{t^{\sigma-\alpha-l}}{(1+t)\left(1+t^{\mu}\right)}\left|x_{n}(t)-x_{0}(t)\right| \longrightarrow 0 \\
\sup _{t \in(0, \infty)} \frac{t^{p+\sigma-\alpha-l}}{1+t^{\mu}}\left|{ }^{c} D_{0^{+}}^{p} x_{n}(t)-{ }^{c} D_{0^{+}}^{p} x_{0}(t)\right| \longrightarrow 0 \\
\text { as } n \longrightarrow \infty
\end{gathered}
$$

Since $f:[0, \infty) \times R^{2} \rightarrow R$ is a Caratheodory function and $\left\{I_{k}\right\}$ is a Caratheodory function sequence, then there exist $M_{r}>0$ and $M_{r k}>0$ such that

$$
\begin{gathered}
\left|f\left(t, x_{n}(t),{ }^{c} D_{0^{+}}^{p} x_{n}(t)\right)\right| \leq M_{r}, \quad t \in[0, \infty), \\
\left|I_{k}\left(t_{k}, x_{n}\left(t_{k}\right)\right)\right| \leq M_{r k}, \quad k=1,2,3, \ldots, \sum_{k=1}^{\infty} M_{r k}<\infty .
\end{gathered}
$$

Notice that

$$
\begin{aligned}
&\left(T x_{n}\right)(t)=\int_{0}^{t} \frac{(t-s)^{\alpha-1}}{\Gamma(\alpha)} q(s) \\
& \times f\left(s, x_{n}(s),{ }^{c} D_{0^{+}}^{p} x_{n}(s)\right) d s \\
&+x_{0}+\sum_{j=1}^{k} I_{j}\left(t_{j}, x_{n}\left(t_{j}\right)\right), \\
& t \in\left(t_{k}, t_{k+1}\right], \quad k=0,1,2, \ldots, \\
&{ }^{c} D_{0^{+}}^{p}\left(T x_{n}\right)(t)= \int_{0}^{t} \frac{(t-s)^{\alpha-p-1}}{\Gamma(\alpha-p)} q(s) \\
& \quad \times f\left(s, x_{n}(s),{ }^{c} D_{0^{+}}^{p} x_{n}(s)\right) d s, \\
& \quad t \in(0, \infty) \backslash\left\{t_{1}, t_{2}, \ldots\right\} .
\end{aligned}
$$

From the inequality

$$
\sum_{j=1}^{\infty}\left|I_{j}\left(t_{j}, x_{n}\left(t_{j}\right)\right)-I_{j}\left(t_{j}, x_{0}\left(t_{j}\right)\right)\right| \leq 2 \sum_{j=1}^{\infty} M_{r k}<\infty,
$$

it follows that there exists $N>0$ for $\epsilon>0$ such that

$$
\sum_{j=N}^{\infty}\left|I_{j}\left(t_{j}, x_{n}\left(t_{j}\right)\right)-I_{j}\left(t_{j}, x_{0}\left(t_{j}\right)\right)\right|<\epsilon .
$$

Since $x \rightarrow I_{k}\left(t_{k},\left(\left(1+t_{k}\right)\left(1+t_{k}^{-\alpha-l}\right) / t_{k}^{-\alpha-l}\right) x\right)(k=1,2, \ldots, N-$ $1)$ is uniformly continuous on $[-r, r]$, there exists $\delta>0$ such that

$$
\begin{aligned}
& \mid I_{k}\left(t_{k}, \frac{\left(1+t_{k}\right)\left(1+t_{k}^{\mu}\right)}{t_{k}^{\sigma-\alpha-l}} x_{1}\right) \\
& \quad-I_{k}\left(t_{k}, \frac{\left(1+t_{k}\right)\left(1+t_{k}^{\mu}\right)}{t_{k}^{\sigma-\alpha-l}} x_{2}\right) \mid<\frac{\epsilon}{N-1}
\end{aligned}
$$

holds for all $x_{1}, x_{2} \in[-r, r]$ with $\left|x_{1}-x_{2}\right|<\delta, k=1$, $2, \ldots, N-1$. From (54), there exists $N_{1}>N$ such that

$$
\begin{gathered}
\frac{t^{\sigma-\alpha-l}}{(1+t)\left(1+t^{\mu}\right)}\left|x_{n}(t)-x_{0}(t)\right|<\delta, \\
t \in(0, \infty), n>N_{1}, \\
\frac{t^{p+\sigma-\alpha-l}}{1+t^{\mu}}\left|{ }^{c} D_{0^{+}}^{p} x_{n}(t)-{ }^{c} D_{0^{+}}^{p} x_{0}(t)\right|<\delta, \\
t \in(0, \infty), \quad n>N_{1} .
\end{gathered}
$$


Hence,

$$
\begin{aligned}
& \sum_{j=1}^{N-1}\left|I_{j}\left(t_{j}, x_{n}\left(t_{j}\right)\right)-I_{j}\left(t_{j}, x_{0}\left(t_{j}\right)\right)\right| \\
& =\sum_{j=1}^{N-1} \mid I_{j}\left(t_{j}, \frac{\left(1+t_{j}\right)\left(1+t_{j}^{\mu}\right)}{t_{j}^{\sigma-\alpha-l}}\right. \\
& \left.\times \frac{t_{j}^{\sigma-\alpha-l}}{\left(1+t_{j}\right)\left(1+t_{j}^{\mu}\right)} x_{n}\left(t_{j}\right)\right) \\
& -I_{j}\left(t_{j}, \frac{\left(1+t_{j}\right)\left(1+t_{j}^{\mu}\right)}{t_{j}^{\sigma-\alpha-l}}\right. \\
& \left.\times \frac{t_{j}^{\sigma-\alpha-l}}{\left(1+t_{j}\right)\left(1+t_{j}^{\mu}\right)} x_{0}\left(t_{j}\right)\right) \\
& <(N-1) \frac{\epsilon}{N-1}=\epsilon, \quad n>N_{1} \text {. }
\end{aligned}
$$

Since

$$
\begin{aligned}
& \frac{t^{\sigma-\alpha-l}}{(1+t)\left(1+t^{\mu}\right)} \\
& \quad \times \int_{0}^{t} \frac{(t-s)^{\alpha-1}}{\Gamma(\alpha)} \mid q(s) f\left(s, x_{n}(s),{ }^{c} D_{0^{+}}^{p} x_{n}(s)\right) \\
& \quad-q(s) f\left(s, x_{0}(s),{ }^{c} D_{0^{+}}^{p} x_{0}(s)\right) \mid d s \\
& \leq 2 M_{r} \frac{t^{\sigma-\alpha-l}}{(1+t)\left(1+t^{\mu}\right)} \int_{0}^{t} \frac{(t-s)^{\alpha-1}}{\Gamma(\alpha)} s^{l} d s \\
& \leq 2 M_{r} \frac{t^{\sigma}}{(1+t)\left(1+t^{\mu}\right)} \int_{0}^{1} \frac{(1-w)^{\alpha-1}}{\Gamma(\alpha)} w^{l} d w \longrightarrow 0
\end{aligned}
$$

therefore, we can find $L>0$ such that

$$
\begin{aligned}
& \frac{t^{\sigma-\alpha-l}}{(1+t)\left(1+t^{\mu}\right)} \\
& \times \int_{0}^{t} \frac{(t-s)^{\alpha-1}}{\Gamma(\alpha)} \\
& \quad \times \mid q(s) f\left(s, x_{n}(s),{ }^{c} D_{0^{+}}^{p} x_{n}(s)\right) \\
& \quad-q(s) f\left(s, x_{0}(s),{ }^{c} D_{0^{+}}^{p} x_{0}(s)\right) \mid d s<\epsilon
\end{aligned}
$$

holds for all $t>L, n=1,2, \ldots$. that

As $f$ is a Caratheodory function, there exists $\delta_{1}>0$ such

$$
\begin{aligned}
& \mid f\left(t, \frac{t^{\sigma-\alpha-l}}{(1+t)\left(1+t^{\mu}\right)} u_{1}, \frac{t^{p+\sigma-\alpha-l}}{1+t^{\mu}} v_{1}\right) \\
& \quad-f\left(t, \frac{t^{\sigma-\alpha-l}}{(1+t)\left(1+t^{\mu}\right)} u_{2}, \frac{t^{p+\sigma-\alpha-l}}{1+t^{\mu}} v_{2}\right) \mid<\epsilon
\end{aligned}
$$

holds for all $t \in[0, L]$ and $u_{1}, u_{2}, v_{1}, v_{2} \in[-r, r]$ with $\mid u_{1}-$ $u_{2}\left|<\delta_{1},\right| v_{1}-v_{2} \mid<\delta_{1}$. From (54), there exists $N_{2}>N>N_{1}$ such that

$$
\begin{array}{r}
\frac{t^{\sigma-\alpha-l}}{(1+t)\left(1+t^{\mu}\right)}\left|x_{n}(t)-x_{0}(t)\right|<\delta_{1}, \\
t \in(0, \infty), \quad n>N_{2}, \\
\frac{t^{p+\sigma-\alpha-l}}{1+t^{\mu}}\left|{ }^{c} D_{0^{+}}^{p} x_{n}(t)-{ }^{c} D_{0^{+}}^{p} x_{0}(t)\right|<\delta_{1}, \\
t \in(0, \infty), \quad n>N_{2} .
\end{array}
$$

So, for $t \in[0, L]$, we have

$$
\begin{aligned}
& \frac{t^{\sigma-\alpha-l}}{(1+t)\left(1+t^{\mu}\right)} \\
& \times \int_{0}^{t} \frac{(t-s)^{\alpha-1}}{\Gamma(\alpha)} \\
& \times \mid q(s) f\left(s, x_{n}(s),{ }^{c} D_{0^{+}}^{p} x_{n}(s)\right) \\
& -q(s) f\left(s, x_{0}(s),{ }^{c} D_{0^{+}}^{p} x_{0}(s)\right) \mid d s \\
& =\frac{t^{\sigma-\alpha-l}}{(1+t)\left(1+t^{\mu}\right)} \\
& \times \int_{0}^{t} \frac{(t-s)^{\alpha-1}}{\Gamma(\alpha)} \\
& \times \mid q(s) f\left(s, \frac{(1+s)\left(1+s^{\mu}\right)}{s^{\sigma-\alpha-l}}\right. \\
& \times \frac{s^{\sigma-\alpha-l}}{(1+s)\left(1+s^{\mu}\right)} x_{n}(s), \\
& \frac{1+s^{\mu}}{s^{p+\sigma-\alpha-l}} \frac{s^{p+\sigma-\alpha-l}}{1+s^{\mu}} \\
& \left.\times{ }^{c} D_{0^{+}}^{p} x_{n}(s)\right) \\
& -q(s) f\left(s, \frac{(1+s)\left(1+s^{\mu}\right)}{s^{\sigma-\alpha-l}}\right. \\
& \times \frac{s^{\sigma-\alpha-l}}{(1+s)\left(1+s^{\mu}\right)} x_{0}(s), \\
& \frac{1+s^{\mu}}{s^{p+\sigma-\alpha-l}} \frac{s^{p+\sigma-\alpha-l}}{1+s^{\mu}} \\
& \left.\times{ }^{c} D_{0^{+}}^{p} x_{0}(s)\right) \mid d s \\
& \leq \frac{t^{\sigma-\alpha-l}}{(1+t)\left(1+t^{\mu}\right)} \int_{0}^{t} \frac{(t-s)^{\alpha-1}}{\Gamma(\alpha)} s^{l} \epsilon d s
\end{aligned}
$$




$$
\begin{aligned}
& =\epsilon \frac{t^{\sigma}}{(1+t)\left(1+t^{\mu}\right)} \int_{0}^{1} \frac{(1-w)^{\alpha-1}}{\Gamma(\alpha)} w^{l} d w \\
& \leq M_{\sigma, \mu} \frac{\mathbf{B}(\alpha, l+1)}{\Gamma(\alpha)} \epsilon, \quad n>N_{2}, t \in[0, L] .
\end{aligned}
$$

Consequently, for all $n>N_{2}, t \in[0, \infty)$, we get

$$
\begin{aligned}
& \frac{t^{\sigma-\alpha-l}}{(1+t)\left(1+t^{\mu}\right)} \\
& \times \int_{0}^{t} \frac{(t-s)^{\alpha-1}}{\Gamma(\alpha)} \\
& \quad \times \mid q(s) f\left(s, x_{n}(s),{ }^{c} D_{0^{+}}^{p} x_{n}(s)\right) \\
& \quad-q(s) f\left(s, x_{0}(s),{ }^{c} D_{0^{+}}^{p} x_{0}(s)\right) \mid d s \\
& <\epsilon+M_{\sigma, \mu} \frac{\mathbf{B}(\alpha, l+1)}{\Gamma(\alpha)} \epsilon .
\end{aligned}
$$

In particular, for $t \in\left(t_{k}, t_{k+1}\right]$, we find that

$$
\begin{aligned}
& \frac{t^{\sigma-\alpha-l}}{(1+t)\left(1+t^{\mu}\right)}\left|\left(T x_{n}\right)(t)-\left(T x_{0}\right)(t)\right| \\
& \leq \frac{t^{\sigma-\alpha-l}}{(1+t)\left(1+t^{\mu}\right)} \\
& \quad \times \int_{0}^{t} \frac{(t-s)^{\alpha-1}}{\Gamma(\alpha)} \\
& \quad \times \mid f\left(s, x_{n}(s),{ }^{c} D_{0^{+}}^{p} x_{n}(s)\right) \\
& \quad+\sum_{j=1}^{k}\left|I_{j}\left(t_{j}, x_{n}\left(t_{j}\right)\right)-I_{j}\left(t_{j}, x_{0}\left(t_{j}\right)\right)\right| \\
& \leq \epsilon+\frac{\mathbf{B}(\alpha, l+1)}{\Gamma(\alpha)} \epsilon \\
& \quad+\sum_{j=1}^{N-1}\left|I_{j}\left(t_{j}, x_{n}\left(t_{j}\right)\right)-I_{j}\left(t_{j}, x_{0}\left(t_{j}\right)\right)\right| \\
& +\sum_{j=N}^{\infty}\left|I_{j}\left(t_{j}, x_{n}\left(t_{j}\right)\right)-I_{j}\left(t_{j}, x_{0}\left(t_{j}\right)\right)\right| \\
& <3 \epsilon+M_{\sigma, \mu} \frac{\mathbf{B}(\alpha, l+1)}{\Gamma(\alpha)} \epsilon, \quad n>N_{2} .
\end{aligned}
$$

Thus, it follows that

$$
\begin{aligned}
\sup _{t \in(0, \infty)} & \frac{t^{\sigma-\alpha-l}}{(1+t)\left(1+t^{\mu}\right)} \\
& \quad \times\left|\left(T x_{n}\right)(t)-\left(T x_{0}\right)(t)\right| \longrightarrow 0 \quad \text { as } n \longrightarrow \infty .
\end{aligned}
$$

Similarly, it can be shown that

$$
\sup _{t \in(0, \infty)} \frac{t^{p+\sigma-\alpha-l}}{1+t^{\mu}}\left|{ }^{c} D_{0^{+}}\left(T x_{n}\right)(t)-^{c} D_{0^{+}}\left(T x_{0}\right)(t)\right| \longrightarrow 0
$$

From (69) and (70), we conclude that $\lim _{n \rightarrow \infty} T x_{n}=T x_{0}$. This implies that $T$ is continuous.

(b) Let us recall that $\Omega \subset X$ is relatively compact if it is bounded, both $\left(t^{\sigma-\alpha-l} /(1+t)\left(1+t^{\mu}\right)\right) \Omega$ and $\left(t^{p+\sigma-\alpha-l} /(1+\right.$ $\left.\left.t^{\mu}\right)\right)^{c} D_{0^{+}}^{p} \Omega$ are equicontinuous on any closed subinterval $[a, b]$ of $\left(t_{k}, t_{k+1}\right](k=0,1,2, \ldots)$ and equiconvergent at $t=$ $t_{k}(k=0,1,2, \ldots)$, and $t=\infty$.

Let $W \subset X$ be a nonempty bounded set. To prove that $T$ is completely continuous, we need to prove that $T W$ is bounded, $T W$ is equicontinuous on finite closed sub-interval on $\left(t_{k}, t_{k+1}\right](k=0,1,2, \ldots), T W$ is equiconvergent at $t=$ $t_{k}(k=0,1,2, \ldots)$, and $T W$ is equiconvergent at $t=\infty$.

Since $W$ is bounded, therefore, (49), (50), and (51) hold for $x \in W$. Following the method of proof for Lemma 8, it can easily be shown that $T W$ is bounded.

Next we show that $T W$ is equicontinuous on finite closed sub-interval on $\left(t_{k}, t_{k+1}\right](k=0,1,2, \ldots)$.

For $[a, b] \subset\left(t_{k}, t_{k+1}\right]$ with $s_{1}, s_{2} \in[a, b]$ with $s_{1}<s_{2}$ and $x \in W$, we have

$$
\begin{aligned}
\left.\mid \frac{s_{1}^{\sigma-\alpha-l}}{(1+} s_{1}\right)\left(1+s_{1}^{\mu}\right) \\
\quad \times \int_{0}^{s_{1}} \frac{\left(s_{1}-s\right)^{\alpha-1}}{\Gamma(\alpha)} q(s) f\left(s, x(s),{ }^{c} D_{0^{+}}^{p} x(s)\right) d s \\
\quad-\frac{s_{2}^{\sigma-\alpha-l}}{\left(1+s_{2}\right)\left(1+s_{2}^{\mu}\right)} \\
\quad \times \int_{0}^{s_{2}} \frac{\left(s_{2}-s\right)^{\alpha-1}}{\Gamma(\alpha)} q(s) f\left(s, x(s),{ }^{c} D_{0^{+}}^{p} x(s)\right) d s \mid \\
\leq\left|\frac{s_{1}^{\sigma-\alpha-l}}{\left(1+s_{1}\right)\left(1+s_{1}^{\mu}\right)}-\frac{s_{2}^{\sigma-\alpha-l}}{\left(1+s_{2}\right)\left(1+s_{2}^{\mu}\right)}\right| \\
\quad \times \int_{0}^{s_{2}} \frac{\left(s_{2}-s\right)^{\alpha-1}}{\Gamma(\alpha)}\left|q(s) f\left(s, x(s),{ }^{c} D_{0^{+}}^{p} x(s)\right)\right| d s \\
\quad+\frac{s_{1}^{\sigma-\alpha-l}}{\left(1+s_{1}\right)\left(1+s_{1}^{\mu}\right)} \\
\quad \times \int_{s_{1}}^{s_{2}} \frac{\left(s_{2}-s\right)^{\alpha-1}}{\Gamma(\alpha)}\left|q(s) f\left(s, x(s),{ }^{c} D_{0^{+}}^{p} x(s)\right)\right| d s \\
\quad+\frac{s_{1}^{\sigma-\alpha-l}}{\left(1+s_{1}\right)\left(1+s_{1}^{\mu}\right)}
\end{aligned}
$$




$$
\begin{aligned}
& \times \int_{0}^{s_{1}} \frac{\left|\left(s_{1}-s\right)^{\alpha-1}-\left(s_{2}-s\right)^{\alpha-1}\right|}{\Gamma(\alpha)} \\
& \times\left|q(s) f\left(s, x(s),{ }^{c} D_{0^{+}}^{p} x(s)\right)\right| d s \\
& \leq\left|\frac{s_{1}^{\sigma-\alpha-l}}{\left(1+s_{1}\right)\left(1+s_{1}^{\mu}\right)}-\frac{s_{2}^{\sigma-\alpha-l}}{\left(1+s_{2}\right)\left(1+s_{2}^{\mu}\right)}\right| \\
& \times M_{r} \int_{0}^{s_{2}} \frac{\left(s_{2}-s\right)^{\alpha-1}}{\Gamma(\alpha)} s^{l} d s \\
& +\frac{s_{1}^{\sigma-\alpha-l}}{\left(1+s_{1}\right)\left(1+s_{1}^{\mu}\right)} M_{r} \int_{s_{1}}^{s_{2}} \frac{\left(s_{2}-s\right)^{\alpha-1}}{\Gamma(\alpha)} s^{l} d s \\
& +M_{r} \frac{s_{1}^{\sigma-\alpha-l}}{\left(1+s_{1}\right)\left(1+s_{1}^{\mu}\right)} \\
& \times \int_{0}^{s_{1}} \frac{\left|\left(s_{1}-s\right)^{\alpha-1}-\left(s_{2}-s\right)^{\alpha-1}\right|}{\Gamma(\alpha)} s^{l} d s \\
& =\left|\frac{s_{1}^{\sigma-\alpha-l}}{\left(1+s_{1}\right)\left(1+s_{1}^{\mu}\right)}-\frac{s_{2}^{\sigma-\alpha-l}}{\left(1+s_{2}\right)\left(1+s_{2}^{\mu}\right)}\right| \\
& \times s_{2}^{\alpha+l} M_{r} \int_{0}^{1} \frac{(1-w)^{\alpha-1}}{\Gamma(\alpha)} w^{l} d w \\
& +\frac{s_{1}^{\sigma-\alpha-l}}{\left(1+s_{1}\right)\left(1+s_{1}^{\mu}\right)} M_{r} s_{2}^{\alpha+l} \\
& \times \int_{s_{1} / s_{2}}^{1} \frac{(1-w)^{\alpha-1}}{\Gamma(\alpha)} w^{l} d w \\
& +M_{r} \frac{s_{1}^{\sigma-\alpha-l}}{\left(1+s_{1}\right)\left(1+s_{1}^{\mu}\right)} \\
& \times \int_{0}^{s_{1}} \frac{\left(s_{1}-s\right)^{\alpha-1}-\left(s_{2}-s\right)^{\alpha-1}}{\Gamma(\alpha)} s^{l} d s \\
& \leq\left|\frac{s_{1}^{\sigma-\alpha-l}}{\left(1+s_{1}\right)\left(1+s_{1}^{\mu}\right)}-\frac{s_{2}^{\sigma-\alpha-l}}{\left(1+s_{2}\right)\left(1+s_{2}^{\mu}\right)}\right| \\
& \times s_{2}^{\alpha+l} M_{r} \int_{0}^{1} \frac{(1-w)^{\alpha-1}}{\Gamma(\alpha)} w^{l} d w \\
& +M_{r} \max \left\{a^{\alpha+l}, b^{\alpha+l}\right\} \int_{s_{1} / s_{2}}^{1} \frac{(1-w)^{\alpha-1}}{\Gamma(\alpha)} w^{l} d w \\
& +M_{r}\left[s_{1}^{\alpha+l} \int_{0}^{1} \frac{(1-w)^{\alpha-1}}{\Gamma(\alpha)} w^{l} d w\right. \\
& \left.-s_{2}^{\alpha+l} \int_{0}^{s_{1} / s_{2}} \frac{(1-w)^{\alpha-1}}{\Gamma(\alpha)} w^{l} d w\right] \\
& \leq\left|\frac{s_{1}^{\sigma-\alpha-l}}{\left(1+s_{1}\right)\left(1+s_{1}^{\mu}\right)}-\frac{s_{2}^{\sigma-\alpha-l}}{\left(1+s_{2}\right)\left(1+s_{2}^{\mu}\right)}\right| \\
& \times \max \left\{a^{\alpha+l}, b^{\alpha+l}\right\} M_{r} \int_{0}^{1} \frac{(1-w)^{\alpha-1}}{\Gamma(\alpha)} w^{l} d w
\end{aligned}
$$

$$
\begin{aligned}
& +M_{r} \max \left\{a^{\alpha+l}, b^{\alpha+l}\right\} \int_{s_{1} / s_{2}}^{1} \frac{(1-w)^{\alpha-1}}{\Gamma(\alpha)} w^{l} d w \\
& +M_{r}\left|s_{1}^{\alpha+l}-s_{2}^{\alpha+l}\right| \int_{0}^{1} \frac{(1-w)^{\alpha-1}}{\Gamma(\alpha)} w^{l} d w \\
& +\max \left\{a^{\alpha+l}, b^{\alpha+l}\right\} \int_{s_{1} / s_{2}}^{1} \frac{(1-w)^{\alpha-1}}{\Gamma(\alpha)} w^{l} d w
\end{aligned}
$$$$
\longrightarrow 0
$$

uniformly as $s_{1} \longrightarrow s_{2}$ with $s_{1}, s_{2} \in[a, b] \subset\left(t_{k}, t_{k+1}\right]$.

So

$$
\begin{aligned}
& \left|\frac{s_{1}^{\sigma-\alpha-l}}{\left(1+s_{1}\right)\left(1+s_{1}^{\mu}\right)}(T x)\left(s_{1}\right)-\frac{s_{2}^{\sigma-\alpha-l}}{\left(1+s_{2}\right)\left(1+s_{2}^{\mu}\right)}(T x)\left(s_{2}\right)\right| \\
& =\mid \frac{s_{1}^{\sigma-\alpha-l}}{\left(1+s_{1}\right)\left(1+s_{1}^{\mu}\right)} \int_{0}^{s_{1}} \frac{\left(s_{1}-s\right)^{\alpha-1}}{\Gamma(\alpha)} q(s) \\
& \times f\left(s, x(s),{ }^{c} D_{0^{+}}^{p} x(s)\right) d s \\
& -\frac{s_{2}^{\sigma-\alpha-l}}{\left(1+s_{2}\right)\left(1+s_{2}^{\mu}\right)} \int_{0}^{s_{2}} \frac{\left(s_{2}-s\right)^{\alpha-1}}{\Gamma(\alpha)} q(s) \\
& \times f\left(s, x(s),{ }^{c} D_{0^{+}}^{p} x(s)\right) d s \\
& +\left|x_{0}\right|\left|\frac{s_{1}^{\sigma-\alpha-l}}{\left(1+s_{1}\right)\left(1+s_{1}^{\mu}\right)}-\frac{s_{2}^{\sigma-\alpha-l}}{\left(1+s_{2}\right)\left(1+s_{2}^{\mu}\right)}\right| \\
& +\left|\frac{s_{1}^{\sigma-\alpha-l}}{\left(1+s_{1}\right)\left(1+s_{1}^{\mu}\right)}-\frac{s_{2}^{\sigma-\alpha-l}}{\left(1+s_{2}\right)\left(1+s_{2}^{\mu}\right)}\right| \\
& \times \sum_{j=1}^{k}\left|I_{j}\left(t_{j}, x\left(t_{j}\right)\right)\right| \\
& \leq \mid \frac{s_{1}^{\sigma-\alpha-l}}{\left(1+s_{1}\right)\left(1+s_{1}^{\mu}\right)} \int_{0}^{s_{1}} \frac{\left(s_{1}-s\right)^{\alpha-1}}{\Gamma(\alpha)} q(s) \\
& \times f\left(s, x(s),{ }^{c} D_{0^{+}}^{p} x(s)\right) d s \\
& -\frac{s_{2}^{\sigma-\alpha-l}}{\left(1+s_{2}\right)\left(1+s_{2}^{\mu}\right)} \int_{0}^{s_{2}} \frac{\left(s_{2}-s\right)^{\alpha-1}}{\Gamma(\alpha)} q(s) \\
& \times f\left(s, x(s),{ }^{c} D_{0^{+}}^{p} x(s)\right) d s \\
& +\left|x_{0}\right|\left|\frac{s_{1}^{\sigma-\alpha-l}}{\left(1+s_{1}\right)\left(1+s_{1}^{\mu}\right)}-\frac{s_{2}^{\sigma-\alpha-l}}{\left(1+s_{2}\right)\left(1+s_{2}^{\mu}\right)}\right| \\
& +\left|\frac{s_{1}^{\sigma-\alpha-l}}{\left(1+s_{1}\right)\left(1+s_{1}^{\mu}\right)}-\frac{s_{2}^{\sigma-\alpha-l}}{\left(1+s_{2}\right)\left(1+s_{2}^{\mu}\right)}\right| \sum_{j=1}^{\infty} M_{r k} \\
& \longrightarrow 0
\end{aligned}
$$

uniformly as $s_{1} \longrightarrow s_{2}$ with $s_{1}, s_{2} \in[a, b] \subset\left(t_{k}, t_{k+1}\right]$. 
Thus,

$$
\begin{aligned}
& \mid \frac{s_{1}^{\sigma-\alpha-l}}{\left(1+s_{1}\right)\left(1+s_{1}^{\mu}\right)}(T x)\left(s_{1}\right) \\
& -\frac{s_{2}^{\sigma-\alpha-l}}{\left(1+s_{2}\right)\left(1+s_{2}^{\mu}\right)}(T x)\left(s_{2}\right) \mid \longrightarrow 0
\end{aligned}
$$

uniformly as $s_{1} \rightarrow s_{2}$ with $s_{1}, s_{2} \in[a, b] \subset\left(t_{k}, t_{k+1}\right]$. Similarly, we have

$$
\begin{gathered}
\left|\frac{s_{1}^{p+\sigma-\alpha-l}}{1+s_{1}^{\mu}}{ }^{c} D_{0^{+}}^{p}(T x)\left(s_{1}\right)-\frac{s_{2}^{p+\sigma-\alpha-l}}{1+s_{2}^{\mu}}{ }^{c} D_{0^{+}}^{p}(T x)\left(s_{2}\right)\right| \\
=\mid \frac{s_{1}^{p+\sigma-\alpha-l}}{1+s_{1}^{\mu}} \int_{0}^{s_{1}} \frac{\left(s_{1}-s\right)^{\alpha-p-1}}{\Gamma(\alpha-p)} q(s) \\
\quad \times f\left(s, x(s),{ }^{c} D_{0^{+}}^{p} x(s)\right) d s \\
-\frac{s_{2}^{p+\sigma-\alpha-l}}{1+s_{2}^{\mu}} \int_{0}^{s_{2}} \frac{\left(s_{2}-s\right)^{\alpha-p-1}}{\Gamma(\alpha-p)} q(s) \\
\times f\left(s, x(s),{ }^{c} D_{0^{+}}^{p} x(s)\right) d s \mid
\end{gathered}
$$

$\longrightarrow 0$

uniformly as $s_{1} \longrightarrow s_{2}$ with $s_{1}, s_{2} \in[a, b] \subset\left(t_{k}, t_{k+1}\right]$.

From (73) and (74), we conclude that $T W$ is equicontinuous on finite closed interval on $\left(t_{k}, t_{k+1}\right]$.

Now wee prove that $T W$ is equiconvergent as $t \rightarrow$ $t_{k}^{+}(k=0,1,2, \ldots)$. For $\mu>\sigma>0$, we find that

$$
\begin{aligned}
& \frac{t^{\sigma-\alpha-l}}{(1+t)\left(1+t^{\mu}\right)}\left|(T x)(t)-x_{0}\right| \\
& \leq \frac{t^{\sigma-\alpha-l}}{(1+t)\left(1+t^{\mu}\right)} \\
& \quad \times \int_{0}^{t} \frac{(t-s)^{\alpha-1}}{\Gamma(\alpha)} q(s) \\
& \quad \times f\left(s, x(s),^{c} D_{0^{+}}^{p} x(s)\right) d s \\
& \leq \frac{M_{r} t^{\sigma}}{(1+t)\left(1+t^{\mu}\right)} \int_{0}^{1} \frac{(1-w)^{\alpha-1}}{\Gamma(\alpha)} w^{l} d w \\
& \longrightarrow 0 \quad \text { uniformly in } W \text { as } t \longrightarrow 0, \\
& \frac{t^{p+\sigma-\alpha-l}}{1+t^{\mu}}\left|{ }^{c} D_{0^{+}}^{p}(T x)(t)\right| \\
& \leq \frac{t^{p+\sigma-\alpha-l}}{1+t^{\mu}}
\end{aligned}
$$

$$
\begin{aligned}
& \times \int_{0}^{t} \frac{(t-s)^{\alpha-p-1}}{\Gamma(\alpha-p)} \\
& \times\left|q(s) f\left(s, x(s),{ }^{c} D_{0^{+}}^{p} x(s)\right)\right| d s \\
\leq & \frac{M_{r} t^{p+\sigma-\alpha-l}}{1+t^{\mu}} \int_{0}^{t} \frac{(t-s)^{\alpha-p-1}}{\Gamma(\alpha-p)} s^{l} d s \\
= & \frac{M_{r} t^{\sigma}}{1+t^{\mu}} \int_{0}^{1} \frac{(1-w)^{\alpha-p-1}}{\Gamma(\alpha-p)} w^{l} d w \\
\longrightarrow & 0 \text { uniformly in } W \text { as } t \longrightarrow 0 .
\end{aligned}
$$

It follows that

$$
\begin{aligned}
& \frac{t^{p+\sigma-\alpha-l}}{1+t^{\mu}}\left|{ }^{c} D_{0^{+}}^{p}(T x)(t)\right| \longrightarrow 0 \\
& \text { uniformly in } W \text { as } t \longrightarrow 0 .
\end{aligned}
$$

From (75), it follows that $T W$ is equiconvergent as $t \rightarrow 0^{+}$.

For $t \rightarrow t_{k}^{+}\left(t \in\left(t_{k}, t_{k+1}\right], k=1,2, \ldots\right)$, we have

$$
\begin{aligned}
& \frac{t^{\sigma-\alpha-l}}{(1+t)\left(1+t^{\mu}\right)} \\
& \times \mid(T x)(t)\left(\int_{0}^{t_{k}} \frac{\left(t_{k}-s\right)^{\alpha-1}}{\Gamma(\alpha)} q(s)\right. \\
& \times f\left(s, x(s),{ }^{c} D_{0^{+}}^{p} x(s)\right) d s \\
& \left.+x_{0}+\sum_{j=1}^{k} I_{j}\left(t_{j}, x\left(t_{j}\right)\right)\right) \\
& \leq \frac{t^{\sigma-\alpha-l}}{(1+t)\left(1+t^{\mu}\right)} \\
& \times \mid \int_{0}^{t} \frac{(t-s)^{\alpha-1}}{\Gamma(\alpha)} q(s) f\left(s, x(s),{ }^{c} D_{0^{+}}^{p} x(s)\right) d s \\
& -\int_{0}^{t_{k}} \frac{\left(t_{k}-s\right)^{\alpha-1}}{\Gamma(\alpha)} q(s) f\left(s, x(s),{ }^{c} D_{0^{+}}^{p} x(s)\right) d s \\
& \leq \frac{t^{\sigma-\alpha-l}}{(1+t)\left(1+t^{\mu}\right)} \\
& \times \int_{t_{k}}^{t} \frac{\left(t_{k}-s\right)^{\alpha-1}}{\Gamma(\alpha)}\left|q(s) f\left(s, x(s),{ }^{c} D_{0^{+}}^{p} x(s)\right)\right| d s \\
& +\frac{t^{\sigma-\alpha-l}}{(1+t)\left(1+t^{\mu}\right)} \\
& \times \int_{0}^{t_{k}} \frac{\left|\left(t_{k}-s\right)^{\alpha-1}-(t-s)^{\alpha-1}\right|}{\Gamma(\alpha)} \\
& \times\left|q(s) f\left(s, x(s),{ }^{c} D_{0^{+}}^{p} x(s)\right)\right| d s
\end{aligned}
$$




$$
\begin{aligned}
& \leq M_{r} \frac{t^{\sigma}}{(1+t)\left(1+t^{\mu}\right)} \\
& \quad \times \int_{t_{k} / t}^{1} \frac{(1-w)^{\alpha-1}}{\Gamma(\alpha)} w^{l} d w \\
& \quad+M_{r} \frac{t^{\sigma-\alpha-l}}{(1+t)\left(1+t^{\mu}\right)} \\
& \quad \times\left[t_{k}^{\alpha+l} \int_{0}^{1} \frac{(1-w)^{\alpha-1}}{\Gamma(\alpha)} w^{l} d w\right. \\
& \left.\quad-\int_{0}^{t_{k} / t} \frac{(1-w)^{\alpha-1}}{\Gamma(\alpha)} w^{l} d w\right]
\end{aligned}
$$

$\longrightarrow 0$ uniformly in $W$ as $t \longrightarrow t_{k}^{+}$,

$$
\begin{aligned}
& \left.\frac{t^{p+\sigma-\alpha-l}}{1+t^{\mu}}\right|^{c} D_{0^{+}}(T x)(t) \\
& -\int_{0}^{t_{k}} \frac{\left(t_{k}-s\right)^{\alpha-p-1}}{\Gamma(\alpha-p)} q(s) \\
& \times f\left(s, x(s),{ }^{c} D_{0^{+}}^{p} x(s)\right) d s \\
& \leq \frac{t^{p+\sigma-\alpha-l}}{1+t^{\mu}} \int_{t_{k}}^{t} \frac{(t-s)^{\alpha-p-1}}{\Gamma(\alpha-p)} \\
& \times\left|q(s) f\left(s, x(s),{ }^{c} D_{0^{+}}^{p} x(s)\right)\right| d s \\
& +\frac{t^{p+\sigma-\alpha-l}}{1+t^{\mu}} \\
& \times \int_{0}^{t_{k}} \frac{\left|\left(t_{k}-s\right)^{\alpha-p-1}-(t-s)^{\alpha-p-1}\right|}{\Gamma(\alpha-p)} \\
& \times\left|q(s) f\left(s, x(s),{ }^{c} D_{0^{+}}^{p} x(s)\right)\right| d s \\
& \leq M_{r} \frac{t^{p+\sigma-\alpha-l}}{1+t^{\mu}} \int_{t_{k}}^{t} \frac{(t-s)^{\alpha-p-1}}{\Gamma(\alpha-p)} s^{l} d s \\
& +M_{r} \frac{t^{p+\sigma-\alpha-l}}{1+t^{\mu}} \\
& \times \int_{0}^{t_{k}} \frac{\left|\left(t_{k}-s\right)^{\alpha-p-1}-(t-s)^{\alpha-p-1}\right|}{\Gamma(\alpha-p)} s^{l} d s \\
& \leq M_{r} M_{\sigma, \mu} \int_{t_{k} / t}^{1} \frac{(1-w)^{\alpha-p-1}}{\Gamma(\alpha-p)} w^{l} d w \\
& +M_{r} \frac{t^{p+\sigma-\alpha-l}}{1+t^{\mu}} \\
& \times\left[t_{k}^{\alpha-p+l} \int_{0}^{1} \frac{(1-w)^{\alpha-p-1}}{\Gamma(\alpha-p)} w^{l} d w\right. \\
& \left.-t^{\alpha-p+l} \int_{0}^{t_{k} / t} \frac{(1-w)^{\alpha-p-1}}{\Gamma(\alpha-p)} w^{l} d w\right]
\end{aligned}
$$

$$
\begin{aligned}
& \leq M_{r} M_{\sigma, \mu} \int_{t_{k} / t}^{1} \frac{(1-w)^{\alpha-p-1}}{\Gamma(\alpha-p)} w^{l} d w \\
& +M_{r} \frac{t^{p+\sigma-\alpha-l}}{1+t^{\mu}} \\
& \times\left[\left(t_{k}^{\alpha-p+l}-t^{\alpha-p+l}\right) \frac{\mathbf{B}(\alpha-p, l+1)}{\Gamma(\alpha-p)}\right. \\
& \left.\quad+t^{\alpha-p+l} \int_{t_{k} / t}^{1} \frac{(1-w)^{\alpha-p-1}}{\Gamma(\alpha-p)} w^{l} d w\right] \\
& \leq M_{r} M_{\sigma, \mu} \int_{t_{k} / t}^{1} \frac{(1-w)^{\alpha-p-1}}{\Gamma(\alpha-p)} w^{l} d w \\
& +M_{r} M_{p+\sigma-\alpha-l, \mu} \\
& \times\left[\left(t_{k}^{\alpha-p+l}-t^{\alpha-p+l}\right) \frac{\mathbf{B}(\alpha-p, l+1)}{\Gamma(\alpha-p)}\right. \\
& \left.\quad+\max \left\{t_{k}^{\alpha-p+l}, t_{k+1}^{\alpha-p+l}\right\} \int_{t_{k} / t}^{1} \frac{(1-w)^{\alpha-p-1}}{\Gamma(\alpha-p)} w^{l} d w\right] \\
& \longrightarrow 0 \quad \text { uniformly in } W \text { as } t \longrightarrow t_{k}^{+},
\end{aligned}
$$

which imply that $T W$ is equiconvergent as $t \rightarrow t_{k}^{+}(k=1$, $2,3, \ldots)$.

Our next task is to show that $T W$ is equiconvergent as $t \rightarrow \infty$. Observe that

$$
\begin{aligned}
& \frac{t^{\sigma-\alpha-l}}{(1+t)\left(1+t^{\mu}\right)} \\
& \quad \times\left|(T x)(t)-\left(x_{0}+\sum_{j=1}^{\infty} I_{j}\left(t_{j}, x\left(t_{j}\right)\right)\right)\right| \\
& \leq \frac{t^{\sigma-\alpha-l}}{(1+t)\left(1+t^{\mu}\right)} \\
& \quad \times \int_{0}^{t} \frac{(t-s)^{\alpha-1}}{\Gamma(\alpha)} q(s) f\left(s, x(s),{ }^{c} D_{0^{+}}^{p} x(s)\right) d s \\
& \leq M_{r} \frac{t^{\sigma}}{(1+t)\left(1+t^{\mu}\right)} \int_{0}^{1} \frac{(1-w)^{\alpha-1}}{\Gamma(\alpha)} w^{l} d w \\
& \longrightarrow 0 \quad \text { uniformly in } \Omega_{1} \text { as } t \longrightarrow \infty, \\
& \frac{t^{p+\sigma-\alpha-l}}{1+}+t^{\mu}||^{c} D_{0^{+}}^{p}(T x)(t) \mid \\
& \leq \frac{t^{p+\sigma-\alpha-l}}{1+t^{\mu}} \int_{0}^{t} \frac{(t-s)^{\alpha-p-1}}{\Gamma(\alpha)} M_{r} s^{l} d s \\
& \longrightarrow 0 \text { uniformly in } W \text { as } t \longrightarrow \infty .
\end{aligned}
$$

Hence, $T W$ is equiconvergent as $t \rightarrow \infty$.

From the above steps, it follows that $T$ is completely continuous. This completes the proof. 
In the sequel, we need the following assumption:

$\left(H_{1}\right) f$ is a Caratheodory function such that

$$
\begin{aligned}
& \left|f\left(t, \frac{(1+t)\left(1+t^{\mu}\right)}{t^{\sigma-\alpha-l}} u_{1}, \frac{1+t^{\mu}}{t^{p+\sigma-\alpha-l}} u_{2}\right)-C\right| \\
& \leq \sum_{i=1}^{m} A_{i}\left|u_{1}\right|^{\delta_{i}}+\sum_{i=1}^{m} B_{i}\left|u_{2}\right|^{\delta_{i}},
\end{aligned}
$$

where $0<\delta_{1}<\delta_{2}<\cdots<\delta_{m}$ and $A_{i}, B_{i}(i=$ $1,2, \ldots, m), C \geq 0$ are real numbers;

$\left(H_{2}\right)\left\{I_{k}\right\}(k=1,2, \ldots)$ is a Caratheodory sequence and there exist numbers $A_{k i} \geq 0(i=1,2, \ldots, m), D_{k} \geq$ $0(k=1,2, \ldots), \delta_{i} \geq 0(i=1,2, \ldots, m)$ such that

$$
\begin{gathered}
\left|I_{k}\left(t_{k}, \frac{\left(1+t_{k}\right)\left(1+t_{k}^{\mu}\right)}{t_{k}^{\sigma-\alpha-l}} u\right)-D_{k}\right| \\
\leq \sum_{i=1}^{m} A_{k i}|u|^{\delta_{i}}
\end{gathered}
$$

$k=1,2,3, \ldots$ holds $\forall t \in(0, \infty), u \in R$.

Furthermore, we set

$$
M_{0}=\max \left\{M_{1}, M_{2}\right\},
$$

where

$$
\begin{array}{r}
M_{1}=\sum_{i=1}^{m}\left[M_{\sigma, \mu} \frac{\mathbf{B}(\alpha, l+1)}{\Gamma(\alpha)}\left[A_{i}+B_{i}\right]\right. \\
\left.+M_{\sigma-\alpha-l, \mu} \sum_{j=1}^{\infty} A_{j i}\right]\|\Psi\|^{\delta_{i}-\delta_{m}}, \\
M_{2}=M_{\sigma, \mu} \frac{\mathbf{B}(\alpha-p, l+1)}{\Gamma(\alpha-p)} \sum_{i=1}^{m}\left[A_{i}+B_{i}\right] .
\end{array}
$$

Theorem 11. Suppose that $\left(H_{1}\right)$ and $\left(H_{2}\right)$ hold. Then IVP (6) has at least one solution $x \in X$ if

$$
\begin{aligned}
& \delta_{m}<1 \quad \text { or } \delta_{m}=1 \quad \text { with } M_{0}<1 \quad \text { or } \\
& \delta_{m}>1 \quad \text { with } \frac{\|\Psi\|^{1-\delta_{m}}\left(\delta_{m}-1\right)^{\delta_{m}-1}}{\delta_{m}^{\delta_{m}}} \geq M_{0} .
\end{aligned}
$$

Proof. Let $X$ be the Banach space as defined in Section 2 and let $T: X \rightarrow X$ be an operator given by (98). In view of Lemma 8 , it follows from the assumptions $\left(H_{1}\right)$ and $\left(H_{2}\right)$ that $T$ is well defined and is completely continuous. Thus, we seek solutions of IVP (6) by finding fixed points of $T$ in $X$.

Let us introduce

$$
\begin{aligned}
\Psi(t)= & C \int_{0}^{t} \frac{(t-s)^{\alpha-1}}{\Gamma(\alpha)} q(s) d s+x_{0} \\
& +\sum_{j=1}^{k} D_{j}, \quad t \in\left(t_{k}, t_{k+1}\right], \quad k=0,1,2, \ldots
\end{aligned}
$$

It is easy to show that $\Psi \in X$. For $r>0$, we define $\bar{\Omega}_{r}=\{x \in$ $X:\|x-\Psi\| \leq r\}$. Then, for $x \in \bar{\Omega}_{r}$, we have

$$
\begin{aligned}
&\|x\|=\max \left\{\sup _{t \in(0, \infty)} \frac{t^{\sigma-\alpha-l}}{(1+t)\left(1+t^{\mu}\right)}|x(t)|,\right. \\
&\left.\sup _{t \in(0, \infty)} \frac{t^{p+\sigma-\alpha-l}}{1+t^{\mu}}\left|{ }^{c} D_{0^{+}}^{p} x(t)\right|\right\} \\
& \leq\|x-\Psi\|+\|\Psi\| \leq r+\|\Psi\| .
\end{aligned}
$$

Using the assumptions $\left(H_{1}\right)$ and $\left(H_{2}\right)$, we find that

$$
\begin{aligned}
& \frac{t^{\sigma-\alpha-l}}{(1+t)\left(1+t^{\mu}\right)}|(T x)(t)-\Psi(t)| \\
& \leq \frac{t^{\sigma-\alpha-l}}{(1+t)\left(1+t^{\mu}\right)} \\
& \quad \times \int_{0}^{t} \frac{(t-s)^{\alpha-1}}{\Gamma(\alpha)}|q(s)| \\
& \quad \times\left|f\left(s, x(s),{ }^{c} D_{0^{+}}^{p} x(s)\right)-C\right| d s
\end{aligned}
$$

$$
\begin{aligned}
& +\frac{t^{\sigma-\alpha-l}}{(1+t)\left(1+t^{\mu}\right)} \sum_{j=1}^{k}\left|I_{j}\left(t_{j}, x\left(t_{j}\right)\right)-D_{j}\right| \\
& \leq \frac{t^{\sigma-\alpha-l}}{(1+t)\left(1+t^{\mu}\right)} \\
& \times \int_{0}^{t} \frac{(t-s)^{\alpha-1}}{\Gamma(\alpha)} s^{l} \\
& \times\left[\sum_{i=1}^{m} A_{i}\left|\frac{t^{\sigma-\alpha-l}}{(1+t)\left(1+t^{\mu}\right)} x(s)\right|^{\delta_{i}}\right. \\
& \left.+\left.\sum_{i=1}^{m} B_{i}\left|\frac{t^{p+\sigma-\alpha-l}}{1+t^{\mu}}{ }^{c} D_{0^{+}}^{p} x(s)\right|\right|^{\delta_{i}}\right] d s \\
& +\frac{t^{\sigma-\alpha-l}}{(1+t)\left(1+t^{\mu}\right)} \\
& \times \sum_{j=1}^{k} \sum_{i=1}^{m} A_{j i}\left|\frac{t_{j}^{\sigma-\alpha-l}}{\left(1+t_{j}\right)\left(1+t_{j}^{\mu}\right)} x\left(t_{j}\right)\right|^{\delta_{i}} \\
& \leq \frac{t^{\sigma}}{(1+t)\left(1+t^{\mu}\right)} \\
& \times \int_{0}^{1} \frac{(1-w)^{\alpha-1}}{\Gamma(\alpha)} w^{l} \\
& \times\left[\sum_{i=1}^{m} A_{i}\|x\|^{\delta_{i}}+\sum_{i=1}^{m} B_{i}\|x\|^{\delta_{i}}\right] d w
\end{aligned}
$$




$$
\begin{aligned}
& +M_{\sigma-\alpha-l} \sum_{j=1}^{k} \sum_{i=1}^{m} A_{j i}\|x\|^{\delta_{i}} \\
& \leq M_{\sigma, \mu} \frac{\mathbf{B}(\alpha, l+1)}{\Gamma(\alpha)} \sum_{i=1}^{m}\left[A_{i}+B_{i}\right]\|x\|^{\delta_{i}} \\
& +M_{\sigma-\alpha-l} \sum_{j=1}^{k} \sum_{i=1}^{m} A_{j i}\|x\|^{\delta_{i}} \\
& =\sum_{i=1}^{m}\left[M_{\sigma, \mu} \frac{\mathbf{B}(\alpha, l+1)}{\Gamma(\alpha)}\left[A_{i}+B_{i}\right]\right. \\
& \left.+M_{\sigma-\alpha-l} \sum_{j=1}^{k} A_{j i}\right]\|x\|^{\delta_{i}} \\
& \leq[r+\|\Psi\|]^{\delta_{m}} \sum_{i=1}^{m}\left[M_{\sigma, \mu} \frac{\mathbf{B}(\alpha, l+1)}{\Gamma(\alpha)}\left[A_{i}+B_{i}\right]\right. \\
& \left.+M_{\sigma-\alpha-l} \sum_{j=1}^{k} A_{j i}\right] \\
& \times[r+\|\Psi\|]^{\delta_{i}-\delta_{m}} \\
& \leq[r+\|\Psi\|]^{\delta_{m}} \\
& \times \sum_{i=1}^{m}\left[M_{\sigma, \mu} \frac{\mathbf{B}(\alpha, l+1)}{\Gamma(\alpha)}\left[A_{i}+B_{i}\right]\right. \\
& \left.+M_{\sigma-\alpha-l, \mu} \sum_{j=1}^{\infty} A_{j i}\right]\|\Psi\|^{\delta_{i}-\delta_{m}} \\
& \leq M_{1}[r+\|\Psi\|]^{\delta_{m}}, \\
& \frac{t^{p+\sigma-\alpha-l}}{1+t^{\mu}}\left|D^{c} D_{0^{+}}^{p}(T x)(t)-{ }^{c} D_{0^{+}}^{p} \Psi(t)\right| \\
& \leq \frac{t^{p+\sigma-\alpha-l}}{1+t^{\mu}} \int_{0}^{t} \frac{(t-s)^{\alpha-p-1}}{\Gamma(\alpha-p)}|q(s)| \\
& \times\left|f\left(s, x(s),{ }^{c} D_{0^{+}}^{p} x(s)\right)-C\right| d s \\
& \leq \frac{t^{p+\sigma-\alpha-l}}{1+t^{\mu}} \\
& \times \int_{0}^{t} \frac{(t-s)^{\alpha-p-1}}{\Gamma(\alpha-p)} s^{l} \\
& \times\left[\sum_{i=1}^{m} A_{i}\left|\frac{t^{\sigma-\alpha-l}}{(1+t)\left(1+t^{\mu}\right)} x(s)\right|^{\delta_{i}}\right. \\
& \left.+\sum_{i=1}^{m} B_{i}\left|\frac{t^{p+\sigma-\alpha-l}}{1+t^{\mu}}{ }^{c} D_{0^{+}}^{p} x(s)\right|^{\delta_{i}}\right] d s
\end{aligned}
$$

$$
\begin{aligned}
\leq & \frac{t^{p+\sigma-\alpha-l}}{1+t^{\mu}} \\
& \times \int_{0}^{t} \frac{(t-s)^{\alpha-p-1}}{\Gamma(\alpha-p)} s^{l} \\
& \times\left[\sum_{i=1}^{m} A_{i}\|x\|^{\delta_{i}}+\sum_{i=1}^{m} B_{i}\|x\|^{\delta_{i}}\right] d s \\
\leq & M_{\sigma, \mu} \frac{\mathbf{B}(\alpha-p, l+1)}{\Gamma(\alpha-p)} \\
& \times\left[\sum_{i=1}^{m} A_{i}\|x\|^{\delta_{i}}+\sum_{i=1}^{m} B_{i}\|x\|^{\delta_{i}}\right] \\
\leq & M_{\sigma, \mu} \frac{\mathbf{B}(\alpha-p, l+1)}{\Gamma(\alpha-p)} \sum_{i=1}^{m}\left[A_{i}+B_{i}\right]\|x\|^{\delta_{i}} \\
\leq & M_{\sigma, \mu} \frac{\mathbf{B}(\alpha-p, l+1)}{\Gamma(\alpha-p)} \\
& \times \sum_{i=1}^{m}\left[A_{i}+B_{i}\right][r+\|\Psi\|]^{\delta_{i}} \\
\leq & {[r+\|\Psi\|]^{\delta_{m}} M_{2} . }
\end{aligned}
$$

Thus, by (81), it follows that

$$
\|T x-\Psi\| \leq[r+\|\Psi\|]^{\delta_{m}} M_{0} .
$$

Next, we have the following cases.

(i) For $\delta_{m}<1$, we can choose $r_{0}>0$ sufficiently large such that $\left[r_{0}+\|\Psi\|\right]^{\delta_{m}} M_{0}<r_{0}$. Let $\Omega_{r_{0}}=\left\{x \in X:\|x\|<r_{0}\right\}$. It is easy to see that $T \bar{\Omega}_{r_{0}} \subset \bar{\Omega}_{r_{0}}$. Then, by Schauder's fixed point theorem, the operator $T$ has a fixed point $x \in \bar{\Omega}_{r_{0}}$, which is a bounded solution of IVP (6).

(ii) In case $\delta_{m}=1$, we choose

$$
r_{0} \geq \frac{\|\Psi\| M_{0}}{1-M_{0}} .
$$

Let $\Omega_{r_{0}}=\left\{x \in X:\|x\|<r_{0}\right\}$. Then it can easily be shown that $T \bar{\Omega}_{r_{0}} \subset \bar{\Omega}_{r_{0}}$. Thus, Schauder's fixed point theorem applies and the operator $T$ has a fixed point $x \in \bar{\Omega}_{r_{0}}$, which is a bounded solution of IVP (6). that

(iii) For $\delta_{m}>1$, we choose $r=r_{0}=\|\Psi\| /\left(\delta_{m}-1\right)$ such

$$
\frac{r_{0}}{\left(r_{0}+\|\Psi\|\right)^{\delta_{m}}}=\frac{\|\Psi\|^{1-\delta_{m}}\left(\delta_{m}-1\right)^{\delta_{m}-1}}{\delta_{m}^{\delta_{m}}} \geq M_{0} .
$$

Let $\Omega_{r_{0}}=\left\{x \in X:\|x\|<r_{0}\right\}$. As before, it is easy to show that $T \bar{\Omega}_{r_{0}} \subset \bar{\Omega}_{r_{0}}$. Then, it follows from Schauder's fixed point theorem that $T$ has a fixed point $x \in \bar{\Omega}_{r_{0}}$, which corresponds to a solution of IVP (6). This completes the proof. 
Theorem 12. Suppose that $\left(H_{1}\right)$ and $\left(H_{2}\right)$ hold with $\delta_{m}=1$. Then IVP (6) has a unique solution $x \in X$ if $M_{0}<1$.

Proof. By Theorem 11, IVP (6) has at least one solution. Let $x_{1}$ and $x_{2}$ be two different solutions of IVP (6). Then $\left\|x_{1}-x_{2}\right\|>$ $0, T x_{1}=x_{1}$, and $T x_{2}=x_{2}$. Employing the method used in the proof of Theorem 11, we find that

$$
\begin{gathered}
\frac{t^{\sigma-\alpha-l}}{(1+t)\left(1+t^{\mu}\right)}\left|\left(T x_{1}\right)(t)-\left(T x_{2}\right)(t)\right| \leq M_{1}\left\|x_{1}-x_{2}\right\|, \\
\frac{t^{p+\sigma-\alpha-l}}{1+t^{\mu}}\left|{ }^{c} D_{0^{+}}^{p}\left(T x_{1}\right)(t)-{ }^{c} D_{0^{+}}^{p}\left(T x_{2}\right)(t)\right| \leq M_{2}\left\|x_{1}-x_{2}\right\| .
\end{gathered}
$$

Thus, $\left\|T x_{1}-T x_{2}\right\| \leq M_{0}\left\|x_{1}-x_{2}\right\|$. On the other hand, by (51), we get

$$
0<\left\|x_{1}-x_{2}\right\|=\left\|T x_{1}-T x_{2}\right\| \leq M_{0}\left\|x_{1}-x_{2}\right\|<\left\|x_{1}-x_{2}\right\|,
$$

which is a contradiction. Hence, IVP (6) has a unique solution $x \in X$ if $M_{0}<1$. This completes the proof.

Next, consider the following IVP:

$$
\begin{gathered}
{ }^{c} D_{0^{+}}^{\alpha} x(t)=q(t) f\left(t, x(t),{ }^{c} D_{0+}^{p} x(t)\right), \quad t \in(0, \infty), \\
x(0)=x_{0}, \\
\Delta x\left(t_{k}\right)=I_{k}\left(t_{k}, x\left(t_{k}\right)\right), \quad k=1,2, \ldots,
\end{gathered}
$$

where $x_{0}, a_{k}(k=1,2, \ldots)$ are constants, $\sum_{k=1}^{\infty}\left|a_{k}\right|$ is convergent, and $f$ is a Caratheodory function; there exists $l \in$ $(-1,-\alpha)$ such that $|q(t)| \leq t^{l}$ for all $t \in(0, \infty)$.

Theorem 13. Assume that the conditions $\left(H_{1}\right)$ and $\left(H_{2}\right)$ hold. Then every solution of (93) tends to $x_{0}+\sum_{k=1}^{\infty} a_{k}$ as $t \rightarrow \infty$ provided that (84) is satisfied.

Proof. By Theorem 11, there exist solutions for IVP (93) satisfying the integral equation

$$
\begin{aligned}
x(t)= & \int_{0}^{t} \frac{(t-s)^{\alpha-1}}{\Gamma(\alpha)} q(s) f\left(s, x(s),{ }^{c} D_{0^{+}}^{p} x(s)\right) d s \\
& +x_{0}+\sum_{j=1}^{k} a_{j}, \quad t \in\left(t_{k}, t_{k+1}\right], k=0,1,2, \ldots
\end{aligned}
$$

Clearly,

$$
\begin{aligned}
\max \left\{\sup _{t \in(0, \infty)} \frac{t^{\sigma-\alpha-l}}{(1+t)\left(1+t^{\mu}\right)}|x(t)|,\right. \\
\left.\sup _{t \in(0, \infty)} \frac{t^{p+\sigma-\alpha-l}}{1+t^{\mu}}\left|{ }^{c} D_{0^{+}}^{p} x(t)\right|\right\} \leq r<\infty .
\end{aligned}
$$

Since $f$ is a Caratheodory function by $\left(H_{1}\right)$, therefore, there exists $M_{r}>0$ such that

$$
\left|f\left(t, x(t),{ }^{c} D_{0^{+}}^{p} x(t)\right)\right| \leq M_{r}, \quad t \in[0, \infty) .
$$

So

$$
\begin{aligned}
& \left|x(t)-\left(x_{0}+\sum_{j=1}^{\infty} a_{j}\right)\right| \\
& =\mid \int_{0}^{t} \frac{(t-s)^{\alpha-1}}{\Gamma(\alpha)} q(s) \\
& \quad \times f\left(s, x(s){ }^{c} D_{0^{+}}^{p} x(s)\right) d s+\sum_{j=k+1}^{\infty} a_{j} \mid \\
& \leq M_{r} \int_{0}^{t} \frac{(t-s)^{\alpha-1} s^{l} d s+\sum_{j=k+1}^{\infty}\left|a_{j}\right|}{\Gamma(\alpha)} M_{r} t^{\alpha+l} \int_{0}^{1} \frac{(1-w)^{\alpha-1}}{\Gamma(\alpha)} w^{l} d w \\
& \quad+\sum_{j=k+1}^{\infty}\left|a_{j}\right| \frac{\text { as } t}{\longrightarrow} \infty .
\end{aligned}
$$

This completes the proof.

\section{Existence of Solutions for an IVP with Multiple Base Points}

In this section, we show the existence for solutions for IVP (7) with multiple base points. Let us introduce an operator $T_{m}$ on $Y$ as

$$
\begin{aligned}
\left(T_{m} x\right)(t)= & \int_{t_{k}}^{t} \frac{(t-s)^{\alpha-1}}{\Gamma(\alpha)} q(s) f\left(s, x(s),{ }^{c} D_{t_{k}^{+}}^{p} x(s)\right) d s \\
& +x_{0}+\sum_{j=1}^{k} I_{j}\left(t_{j}, x\left(t_{j}\right)\right) \\
& +\sum_{j=1}^{k} \int_{t_{j-1}}^{t_{j}} \frac{\left(t_{j}-s\right)^{\alpha-1}}{\Gamma(\alpha)} q(s) \\
& \quad \times f\left(s, x(s),{ }^{c} D_{t_{j-1}^{+}}^{p} x(s)\right) d s, \\
& \quad t \in\left(t_{k}, t_{k+1}\right], \quad k=0,1,2, \ldots
\end{aligned}
$$

Lemma 14. Suppose that $f$ is a Caratheodory function and $\left\{I_{k}\right\}$ is a Caratheodory function sequence and $\lambda_{0}=$ : $\inf _{k=1,2,3, \ldots}\left(t_{k}-t_{k-1}\right)>0$. Then

(i) $T_{m}: Y \rightarrow Y$ is well defined;

(ii) the fixed point of the operator $T_{m}$ coincides with the solution of IVP (7);

(iii) $T_{m}: Y \rightarrow Y$ is completely continuous. 
Proof. (i) For $x \in Y$, we set

$$
\begin{aligned}
r=\max & \left\{\sup _{t \in(0, \infty)} \frac{t^{\sigma-\alpha-l}}{(1+t)\left(1+t^{\mu}\right)}|x(t)|,\right. \\
& \left.\sup _{k=0,1,2, \ldots .} \sup _{t \in\left(t_{k}, t_{k+1}\right]} \frac{t^{p+\sigma-\alpha-l}}{1+t^{\mu}}\left|{ }^{c} D_{t_{k}^{+}}^{p} x(t)\right|\right\}<+\infty .
\end{aligned}
$$

Since $f$ is a Caratheodory function, $\left\{I_{k}\right\}$ is Caratheodory function sequence; there exist positive numbers $\widetilde{M}_{r}>0$ and $M_{r k}>0(k=1,2, \ldots)$ such that

$$
\begin{gathered}
\left|f\left(t, x(t),{ }^{c} D_{t_{k}^{+}}^{p} x(t)\right)\right| \leq \widetilde{M}_{r}, \quad t \in[0, \infty), \\
\left|I_{k}\left(t_{k}, x\left(t_{k}\right)\right)\right| \leq M_{r k}, \quad k=1,2, \ldots, \sum_{k=1}^{\infty} M_{r k}<\infty .
\end{gathered}
$$

It is easy to show that

$$
\begin{array}{r}
\left.T_{m} x\right|_{\left(t_{k}, t_{k+1}\right]} \in C^{0}\left(t_{k}, t_{k+1}\right], \\
\left.{ }^{c} D_{t_{k}^{+}}^{p} T_{m} x\right|_{\left(t_{k}, t_{k+1}\right]} \\
\in C^{0}\left(t_{k}, t_{k+1}\right], \\
k=0,1,2, \ldots
\end{array}
$$

As in Lemma 9, we can show that

$$
\begin{gathered}
\frac{t^{\sigma-\alpha-l}}{(1+t)\left(1+t^{\mu}\right)}\left(T_{m} x\right)(t) \text { is bounded, } \\
\left\{\sup _{t \in\left(t_{k}, t_{k+1}\right]} \frac{t^{p+\sigma-\alpha-l}}{1+t^{\mu}} D_{t_{k}^{+}}^{p}\left(T_{m} x\right)(t)\right\}_{k=0}^{\infty} \text { is bounded. }
\end{gathered}
$$

Hence, $T_{m} x \in Y$. This implies that $T_{m}: Y \rightarrow Y$ is well defined.

(ii) It follows from Lemma 9 that the fixed point of the operator $T_{m}$ coincides with the solution of IVP (7).

(iii) To show that $T_{m}$ is completely continuous, we split the proof into several steps.

Step 1. $T_{m}$ is continuous.

Let $x_{n} \in Y$ with $x_{n} \rightarrow x_{0}$ as $n \rightarrow \infty$. We will prove that $T_{m} x_{n} \rightarrow T_{m} x_{0}$ as $n \rightarrow \infty$. It is easy to see that there exists $r>0$ such that

$$
\begin{aligned}
\max \left\{\sup _{t \in(0, \infty)} \frac{t^{\sigma-\alpha-l}}{(1+t)\left(1+t^{\mu}\right)}\left|x_{n}(t)\right|,\right. & \\
\left.\sup _{k=0,1,2, \ldots} \sup _{t \in\left(t_{k}, t_{k+1}\right]} \frac{t^{p+\sigma-\alpha-l}}{1+t^{\mu}}\left|{ }^{c} D_{t_{k}^{+}}^{p} x_{n}(t)\right|\right\} & \leq r<\infty, \\
n & =0,1,2, \ldots,
\end{aligned}
$$

$$
\begin{aligned}
\sup _{t \in(0, \infty)} \frac{t^{\sigma-\alpha-l}}{(1+t)\left(1+t^{\mu}\right)}\left|x_{n}(t)-x_{0}(t)\right| & \longrightarrow 0 \\
\text { as } n \longrightarrow \infty, & \\
\sup _{k=0,1,2, \ldots} \sup _{t \in\left(t_{k}, t_{k+1}\right]} \frac{t^{p+\sigma-\alpha-l}}{1+t^{\mu}}\left|{ }^{c} D_{t_{k}^{+}}^{p} x_{n}(t)-{ }^{c} D_{t_{k}^{+}}^{p} x_{0}(t)\right| & \longrightarrow 0 \\
\text { as } n & \longrightarrow \infty .
\end{aligned}
$$

As in the proof of Lemma 10,

$$
\sum_{j=1}^{N-1}\left|I_{j}\left(t_{j}, x_{n}\left(t_{j}\right)\right)-I_{j}\left(t_{j}, x_{0}\left(t_{j}\right)\right)\right|<\epsilon, \quad n>N_{1} \text {. }
$$

From $\lambda_{0}=\inf _{k=1,2, \ldots}\left(t_{k}-t_{k-1}\right)>0$, we get $t_{k}>k \lambda_{0}$ for all $k=0,1,2, \ldots$

Since $\sum_{j=K+1}^{\infty}\left(1 / j^{\mu+1-\sigma}\right)$ is convergent, there exists $K>0$ such that

$$
\sum_{j=K+1}^{\infty} \frac{1}{j^{\mu+1-\sigma}}<\epsilon
$$

Then

$$
\begin{aligned}
& \frac{t^{\sigma-\alpha-l}}{(1+t)\left(1+t^{\mu}\right)} \\
& \times \int_{t_{k}}^{t} \frac{(t-s)^{\alpha-1}}{\Gamma(\alpha)} \\
& \times \mid q(s) f\left(s, x_{n}(s),{ }^{c} D_{t_{k}^{+}}^{p} x_{n}(s)\right) \\
& -q(s) f\left(s, x_{0}(s),{ }^{c} D_{t_{k}^{+}}^{p} x_{0}(s)\right) \mid d s \\
& +\frac{t^{\sigma-\alpha-l}}{(1+t)\left(1+t^{\mu}\right)} \\
& \times \sum_{j=K+1}^{k} \int_{t_{j-1}}^{t_{j}} \frac{\left(t_{j}-s\right)^{\alpha-1}}{\Gamma(\alpha)} \\
& \times \mid q(s) f\left(s, x_{n}(s),{ }^{c} D_{t_{k}^{+}}^{p} x_{n}(s)\right) \\
& -q(s) f\left(s, x_{0}(s),{ }^{c} D_{t_{k}^{+}}^{p} x_{0}(s)\right) \mid d s \\
& \leq 2 \widetilde{M}_{r} \frac{t^{\sigma-\alpha-l}}{(1+t)\left(1+t^{\mu}\right)} \int_{t_{k}}^{t} \frac{(t-s)^{\alpha-1}}{\Gamma(\alpha)} s^{l} d s \\
& +2 M_{r} \frac{t^{\sigma-\alpha-l}}{(1+t)\left(1+t^{\mu}\right)} \\
& \times \sum_{j=K+1}^{k} \int_{t_{j-1}}^{t_{j}} \frac{\left(t_{j}-s\right)^{\alpha-1}}{\Gamma(\alpha)} s^{l} d s \\
& \leq 2 \widetilde{M}_{r} \frac{1}{t^{\mu+1-\sigma}} \int_{t_{k} / t}^{1} \frac{(1-w)^{\alpha-1}}{\Gamma(\alpha)} w^{l} d w
\end{aligned}
$$




$$
\begin{aligned}
& +2 \widetilde{M}_{r} \frac{1}{t^{\mu+1-\sigma+\alpha+l}} \\
& \times \sum_{j=K+1}^{k} t_{j}^{\alpha+l} \int_{t_{j-1} / t_{j}}^{1} \frac{(1-w)^{\alpha-1}}{\Gamma(\alpha)} w^{l} d w \\
& \leq 2 \widetilde{M}_{r} \frac{1}{t_{k}^{\mu+1-\sigma}} \int_{0}^{1} \frac{(1-w)^{\alpha-1}}{\Gamma(\alpha)} w^{l} d w \\
& +2 \widetilde{M}_{r} \sum_{j=K+1}^{k} \frac{1}{t_{j}^{\mu+1-\sigma+\alpha+l}} t_{j}^{\alpha+l} \\
& \times \int_{0}^{1} \frac{(1-w)^{\alpha-1}}{\Gamma(\alpha)} w^{l} d w \\
& \leq 4 \widetilde{M}_{r} \sum_{j=K+1}^{k} \frac{1}{t_{j}^{\mu+1-\sigma}} \frac{\mathbf{B}(\alpha, l+1)}{\Gamma(\alpha)} \\
& =4 M_{r} \frac{\mathbf{B}(\alpha, l+1)}{\Gamma(\alpha)} \sum_{j=K+1}^{k} \frac{1}{t_{j}^{\mu+1-\sigma}} \\
& \leq 4 \widetilde{M}_{r} \frac{\mathbf{B}(\alpha, l+1)}{\Gamma(\alpha)} \sum_{j=K+1}^{k} \frac{1}{\left(j \lambda_{0}\right)^{\mu+1-\sigma}} \\
& =4 \widetilde{M}_{r} \frac{\mathbf{B}(\alpha, l+1)}{\Gamma(\alpha)} \frac{1}{\lambda_{0}^{\mu+1-\sigma}} \sum_{j=K+1}^{\infty} \frac{1}{j^{\mu+1-\sigma}} \\
& \leq 4 \widetilde{M}_{r} \frac{\mathbf{B}(\alpha, l+1)}{\Gamma(\alpha)} \frac{1}{\lambda_{0}^{\mu+1-\sigma}} \epsilon .
\end{aligned}
$$

Since $f$ is a Caratheodory function, there exists $\delta_{1}>0$ such that

$$
\begin{aligned}
& \mid f\left(t, \frac{t^{\sigma-\alpha-l}}{(1+t)\left(1+t^{\mu}\right)} u_{1}, \frac{t^{p+\sigma-\alpha-l}}{1+t^{\mu}} v_{1}\right) \\
& \quad-f\left(t, \frac{t^{\sigma-\alpha-l}}{(1+t)\left(1+t^{\mu}\right)} u_{2}, \frac{t^{p+\sigma-\alpha-l}}{1+t^{\mu}} v_{2}\right) \mid \\
& <\frac{\epsilon}{\sum_{j=1}^{K}\left(1 / t_{j}^{\mu+1-\sigma}\right)}
\end{aligned}
$$

holds for all $t \in\left[0, t_{K+1}\right]$ and $u_{1}, u_{2} \in[-r, r]$ with $\left|u_{1}-u_{2}\right|<$ $\delta_{1},\left|v_{1}-v_{2}\right|<\delta_{1}$. From (104), there exists $N_{2}>N_{1}$ such that

$$
\begin{array}{r}
\frac{t^{\sigma-\alpha-l}}{(1+t)\left(1+t^{\mu}\right)}\left|x_{n}(t)-x_{0}(t)\right|<\delta_{1}, \\
t \in(0, \infty), n>N_{2}, \\
\frac{t^{p+\sigma-\alpha-l}}{1+t^{\mu}}\left|{ }^{c} D_{t_{k}^{+}}^{p} x_{n}(t)-{ }^{c} D_{t_{k}^{+}}^{p} x_{0}(t)\right|<\delta_{1}, \\
t \in\left(t_{k}, t_{k+1}\right], \quad n>N_{2} .
\end{array}
$$

So, for $t \in\left[t_{k}, t_{k+1}\right]$, we have

$$
\begin{aligned}
& \frac{t^{\sigma-\alpha-l}}{(1+t)\left(1+t^{\mu}\right)} \\
& \times \sum_{j=1}^{K} \int_{t_{j-1}}^{t_{j}} \frac{\left(t_{j}-s\right)^{\alpha-1}}{\Gamma(\alpha)} \\
& \times \mid q(s) f\left(s, x_{n}(s),{ }^{c} D_{t_{k}^{+}}^{p} x_{n}(s)\right) \\
& -q(s) f\left(s, x_{0}(s),{ }^{c} D_{t_{k}^{+}}^{p} x_{0}(s)\right) \mid d s \\
& \leq \frac{t^{\sigma-\alpha-l}}{(1+t)\left(1+t^{\mu}\right)} \\
& \times \sum_{j=1}^{K} \int_{t_{j-1}}^{t_{j}} \frac{\left(t_{j}-s\right)^{\alpha-1}}{\Gamma(\alpha)} \\
& \times s^{l} \frac{\epsilon}{\sum_{j=1}^{K}\left(1 / t_{j}^{\mu+1-\sigma}\right)} d s \\
& \leq \frac{\epsilon}{\sum_{j=1}^{K}\left(1 / t_{j}^{\mu+1-\sigma}\right)} \frac{1}{t^{\mu+1-\sigma+\alpha+l}} \\
& \times \sum_{j=1}^{K} t_{j}^{\alpha+l} \int_{t_{j-1} / t_{j}}^{1} \frac{(1-w)^{\alpha-1}}{\Gamma(\alpha)} w^{l} d w \\
& \leq \frac{\epsilon}{\sum_{j=1}^{K}\left(1 / t_{j}^{\mu+1-\sigma}\right)} \\
& \times \sum_{j=1}^{K} \frac{1}{t_{j}^{\mu+1-\sigma}} \int_{0}^{1} \frac{(1-w)^{\alpha-1}}{\Gamma(\alpha)} w^{l} d w \\
& \leq \frac{\mathbf{B}(\alpha, l+1)}{\Gamma(\alpha)} \epsilon, \quad n>N_{2} .
\end{aligned}
$$

Thus, for $t \in\left(t_{k}, t_{k+1}\right](k=0,1,2, \ldots)$ with $n>N_{2}$, we have

$$
\begin{aligned}
& \frac{t^{\sigma-\alpha-l}}{(1+t)\left(1+t^{\mu}\right)}\left|\left(T_{m} x_{n}\right)(t)-\left(T_{m} x_{0}\right)(t)\right| \\
& \leq \frac{t^{\sigma-\alpha-l}}{(1+t)\left(1+t^{\mu}\right)} \\
& \quad \times \int_{t_{k}}^{t} \frac{(t-s)^{\alpha-1}}{\Gamma(\alpha)} \\
& \quad \times \mid f\left(s, x_{n}(s),{ }^{c} D_{t_{k}^{+}}^{p} x_{n}(s)\right) \\
& \quad-f\left(s, x_{0}(s),{ }^{c} D_{t_{k}^{+}}^{p} x_{0}(s)\right) \mid d s
\end{aligned}
$$




$$
\begin{aligned}
& +\sum_{j=1}^{\infty}\left|I_{j}\left(t_{j}, x_{n}\left(t_{j}\right)\right)-I_{j}\left(t_{j}, x_{0}\left(t_{j}\right)\right)\right| \\
& +\frac{t^{\sigma-\alpha-l}}{(1+t)\left(1+t^{\mu}\right)} \\
& \times \sum_{j=1}^{k} \int_{t_{j-1}}^{t_{j}} \frac{\left(t_{j}-s\right)^{\alpha-1}}{\Gamma(\alpha)}|q(s)| \\
& \times \mid f\left(s, x_{n}(s),{ }^{c} D_{t_{j-1}^{+}}^{p} x_{n}(s)\right) \\
& \leq 3 \epsilon+4 M_{r} \frac{\mathbf{B}(\alpha, l+1)}{\Gamma(\alpha)} \frac{1}{\lambda_{0}^{\mu+1-\sigma}} \epsilon \\
& \left.+\frac{\mathbf{B}(\alpha, l+1)}{\Gamma(\alpha)} \epsilon, \quad n>x_{0}(s), D_{t_{j-1}}^{p} x_{0}(s)\right) \mid d s
\end{aligned}
$$

In consequence,

$$
\begin{aligned}
& \sup _{k=0,1,2, \ldots} \sup _{t \in\left(t_{k}, t_{k+1}\right]} \frac{t^{\sigma-\alpha-l}}{(1+t)\left(1+t^{\mu}\right)} \\
& \quad \times\left|\left(T_{m} x_{n}\right)(t)-\left(T_{m} x_{0}\right)(t)\right| \longrightarrow 0 \quad \text { as } n \rightarrow \infty .
\end{aligned}
$$

Similarly, we can show that

$$
\begin{aligned}
\sup _{k=0,1,2, \ldots} & \sup _{t \in\left(t_{k}, t_{k+1}\right]} \frac{t^{p+\sigma-\alpha-l}}{1+t^{\mu}} \\
\times\left|{ }^{c} D_{t_{k}^{+}}\left(T_{m} x_{n}\right)(t)-{ }^{c} D_{t_{k}^{+}}\left(T_{m} x_{0}\right)(t)\right| & \longrightarrow 0 \\
\text { as } n & \longrightarrow \infty .
\end{aligned}
$$

From (112) and (113), it follows that $\lim _{n \rightarrow \infty} T_{m} x_{n}=T_{m} x_{0}$ which implies that $T_{m}$ is continuous.

Let $W \subset X$ be a nonempty bounded set. To prove that $T_{m}$ is completely continuous, we need to prove that $T_{m} W$ is bounded, $T_{m} W$ is equicontinuous on finite closed subinterval on $\left(t_{k}, t_{k+1}\right](k=0,1,2 \ldots), T_{m} W$ is equiconvergent at $t=t_{k}(k=0,1,2, \ldots)$, and $T_{m} W$ is equiconvergent at $t=\infty$.

Step 2. As in the proof of Lemma 10, it is easy to show that $T_{m} W$ is bounded.

Step 3. We prove that $T_{m} W$ is equicontinuous on finite closed sub-interval on $\left(t_{k}, t_{k+1}\right](k=0,1,2, \ldots)$. For $[a, b] \subset$ $\left(t_{k}, t_{k+1}\right]$ with $s_{1}, s_{2} \in[a, b]$ with $s_{1}<s_{2}$ and $x \in W$, we have

$$
\begin{aligned}
& \mid \frac{s_{1}^{\sigma-\alpha-l}}{\left(1+s_{1}\right)\left(1+s_{1}^{\mu}\right)} \\
& \quad \times \int_{t_{k}}^{s_{1}} \frac{\left(s_{1}-s\right)^{\alpha-1}}{\Gamma(\alpha)} q(s) \\
& \quad \times f\left(s, x(s),{ }^{c} D_{t_{k}^{+}}^{p} x(s)\right) d s
\end{aligned}
$$

$$
\begin{aligned}
& -\frac{s_{2}^{\sigma-\alpha-l}}{\left(1+s_{2}\right)\left(1+s_{2}^{\mu}\right)} \\
& \times \int_{t_{k}}^{s_{2}} \frac{\left(s_{2}-s\right)^{\alpha-1}}{\Gamma(\alpha)} q(s) \\
& \quad \times f\left(s, x(s),{ }^{c} D_{t_{k}^{+}}^{p} x(s)\right) d s \mid \\
& \leq\left|\frac{s_{1}^{\sigma-\alpha-l}}{\left(1+s_{1}\right)\left(1+s_{1}^{\mu}\right)}-\frac{s_{2}^{\sigma-\alpha-l}}{\left(1+s_{2}\right)\left(1+s_{2}^{\mu}\right)}\right| \\
& \quad \times \int_{t_{k}}^{s_{2}} \frac{\left(s_{2}-s\right)^{\alpha-1}}{\Gamma(\alpha)}
\end{aligned}
$$$$
\times\left|q(s) f\left(s, x(s),{ }^{c} D_{t_{k}^{+}}^{p} x(s)\right)\right| d s
$$$$
+\frac{s_{1}^{\sigma-\alpha-l}}{\left(1+s_{1}\right)\left(1+s_{1}^{\mu}\right)}
$$$$
\times \int_{s_{1}}^{s_{2}} \frac{\left(s_{2}-s\right)^{\alpha-1}}{\Gamma(\alpha)}
$$$$
\times\left|q(s) f\left(s, x(s),{ }^{c} D_{t_{k}^{+}}^{p} x(s)\right)\right| d s
$$$$
+\frac{s_{1}^{\sigma-\alpha-l}}{\left(1+s_{1}\right)\left(1+s_{1}^{\mu}\right)}
$$$$
\times \int_{t_{k}}^{s_{1}} \frac{\left|\left(s_{1}-s\right)^{\alpha-1}-\left(s_{2}-s\right)^{\alpha-1}\right|}{\Gamma(\alpha)}
$$

$$
\times\left|q(s) f\left(s, x(s),{ }^{c} D_{t_{k}^{+}}^{p} x(s)\right)\right| d s
$$

$$
\begin{aligned}
\leq & \left|\frac{s_{1}^{\sigma-\alpha-l}}{\left(1+s_{1}\right)\left(1+s_{1}^{\mu}\right)}-\frac{s_{2}^{\sigma-\alpha-l}}{\left(1+s_{2}\right)\left(1+s_{2}^{\mu}\right)}\right| \\
& \times \widetilde{M}_{r} \int_{t_{k}}^{s_{2}} \frac{\left(s_{2}-s\right)^{\alpha-1}}{\Gamma(\alpha)} s^{l} d s
\end{aligned}
$$$$
+\frac{s_{1}^{\sigma-\alpha-l}}{\left(1+s_{1}\right)\left(1+s_{1}^{\mu}\right)} \widetilde{M}_{r} \int_{s_{1}}^{s_{2}} \frac{\left(s_{2}-s\right)^{\alpha-1}}{\Gamma(\alpha)} s^{l} d s
$$$$
+\widetilde{M}_{r} \frac{s_{1}^{\sigma-\alpha-l}}{\left(1+s_{1}\right)\left(1+s_{1}^{\mu}\right)}
$$$$
\times \int_{t_{k}}^{s_{1}} \frac{\left|\left(s_{1}-s\right)^{\alpha-1}-\left(s_{2}-s\right)^{\alpha-1}\right|}{\Gamma(\alpha)} s^{l} d s
$$

$$
\begin{aligned}
& =\left|\frac{s_{1}^{\sigma-\alpha-l}}{\left(1+s_{1}\right)\left(1+s_{1}^{\mu}\right)}-\frac{s_{2}^{\sigma-\alpha-l}}{\left(1+s_{2}\right)\left(1+s_{2}^{\mu}\right)}\right| s_{2}^{\alpha+l} \\
& \quad \times \widetilde{M}_{r} \int_{0}^{1} \frac{(1-w)^{\alpha-1}}{\Gamma(\alpha)} w^{l} d w
\end{aligned}
$$




$$
\begin{aligned}
& +\frac{s_{1}^{\sigma-\alpha-l}}{\left(1+s_{1}\right)\left(1+s_{1}^{\mu}\right)} \widetilde{M}_{r} s_{2}^{\alpha+l} \int_{s_{1} / s_{2}}^{1} \frac{(1-w)^{\alpha-1}}{\Gamma(\alpha)} w^{l} d w \\
& +\widetilde{M}_{r} \frac{s_{1}^{\sigma-\alpha-l}}{\left(1+s_{1}\right)\left(1+s_{1}^{\mu}\right)} \\
& \times \int_{t_{k}}^{s_{1}} \frac{\left(s_{1}-s\right)^{\alpha-1}-\left(s_{2}-s\right)^{\alpha-1}}{\Gamma(\alpha)} s^{l} d s \\
& \leq\left|\frac{s_{1}^{\sigma-\alpha-l}}{\left(1+s_{1}\right)\left(1+s_{1}^{\mu}\right)}-\frac{s_{2}^{\sigma-\alpha-l}}{\left(1+s_{2}\right)\left(1+s_{2}^{\mu}\right)}\right| \\
& \times s_{2}^{\alpha+l} \widetilde{M}_{r} \int_{0}^{1} \frac{(1-w)^{\alpha-1}}{\Gamma(\alpha)} w^{l} d w \\
& +\widetilde{M}_{r} \max \left\{a^{\alpha+l}, b^{\alpha+l}\right\} \int_{s_{1} / s_{2}}^{1} \frac{(1-w)^{\alpha-1}}{\Gamma(\alpha)} w^{l} d w \\
& +\widetilde{M}_{r}\left[s_{1}^{\alpha+l} \int_{0}^{1} \frac{(1-w)^{\alpha-1}}{\Gamma(\alpha)} w^{l} d w\right. \\
& +\widetilde{M}_{r}\left|s_{1}^{\alpha+l}-s_{2}^{\alpha+l}\right| \int_{0}^{1} \frac{(1-w)^{\alpha-1}}{\Gamma(\alpha)} w^{l} d w \\
& +\widetilde{M}_{r} \max \left\{a^{\alpha+l}, b^{\alpha+l}\right\} \int_{s_{1} / s_{2}}^{1} \frac{(1-w)^{\alpha-1}}{\Gamma(\alpha)} w^{l} d w \\
& \leq\left|\frac{s_{2}^{\alpha+l} \int_{0}^{s_{1} / s_{2}} \frac{(1-w)^{\alpha-1}}{\Gamma(\alpha)} w^{l} d w}{\left(1+s_{1}\right)\left(1+s_{1}^{\mu}\right)}-\frac{\left.s_{2}^{\sigma-\alpha-l}\right\}}{\left(1+s_{2}\right)\left(1+s_{2}^{\mu}\right)}\right| \\
& \quad \max \left\{a_{s_{1} / s_{2}}^{\alpha+l}, b^{\alpha+l}\right\} \widetilde{M}_{r} \int_{0}^{1} \frac{(1-w)^{\alpha-1}}{\Gamma(\alpha)} w^{l} d w \\
& \Gamma(\alpha) \\
&
\end{aligned}
$$

uniformly as $s_{1} \longrightarrow s_{2}$ with $s_{1}, s_{2} \in[a, b] \subset\left(t_{k}, t_{k+1}\right]$.

(114)

So

$$
\begin{gathered}
\left|\frac{s_{1}^{\sigma-\alpha-l}}{\left(1+s_{1}\right)\left(1+s_{1}^{\mu}\right)}\left(T_{m} x\right)\left(s_{1}\right)-\frac{s_{2}^{\sigma-\alpha-l}}{\left(1+s_{2}\right)\left(1+s_{2}^{\mu}\right)}\left(T_{m} x\right)\left(s_{2}\right)\right| \\
=\mid \frac{s_{1}^{\sigma-\alpha-l}}{\left(1+s_{1}\right)\left(1+s_{1}^{\mu}\right)} \\
\quad \times \int_{t_{k}}^{s_{1}} \frac{\left(s_{1}-s\right)^{\alpha-1}}{\Gamma(\alpha)} q(s) f\left(s, x(s),{ }^{c} D_{t_{k}^{+}}^{p} x(s)\right) d s \\
\quad-\frac{s_{2}^{\sigma-\alpha-l}}{\left(1+s_{2}\right)\left(1+s_{2}^{\mu}\right)}
\end{gathered}
$$

$$
\begin{aligned}
& \times \int_{t_{k}}^{s_{2}} \frac{\left(s_{2}-s\right)^{\alpha-1}}{\Gamma(\alpha)} q(s) f\left(s, x(s),{ }^{c} D_{t_{k}^{+}}^{p} x(s)\right) d s \mid \\
+ & \left|x_{0}\right|\left|\frac{s_{1}^{\sigma-\alpha-l}}{\left(1+s_{1}\right)\left(1+s_{1}^{\mu}\right)}-\frac{s_{2}^{\sigma-\alpha-l}}{\left(1+s_{2}\right)\left(1+s_{2}^{\mu}\right)}\right| \\
+ & \left|\frac{s_{1}^{\sigma-\alpha-l}}{\left(1+s_{1}\right)\left(1+s_{1}^{\mu}\right)}-\frac{s_{2}^{\sigma-\alpha-l}}{\left(1+s_{2}\right)\left(1+s_{2}^{\mu}\right)}\right| \\
\times & \sum_{j=1}^{k}\left|I_{j}\left(t_{j}, x\left(t_{j}\right)\right)\right| \\
+ & \left|\frac{s_{1}^{\sigma-\alpha-l}}{\left(1+s_{1}\right)\left(1+s_{1}^{\mu}\right)}-\frac{s_{2}^{\sigma-\alpha-l}}{\left(1+s_{2}\right)\left(1+s_{2}^{\mu}\right)}\right| \\
\times & \times \sum_{j=1}^{k} \int_{t_{k-1}}^{t_{k}} \frac{\left(t_{k}-s\right)^{\alpha-1}}{\Gamma(\alpha)}\left|q(s) f\left(s, x(s),{ }^{c} D_{t_{k}^{+}}^{p} x(s)\right)\right| d s
\end{aligned}
$$$$
\leq \mid \frac{s_{1}^{\sigma-\alpha-l}}{\left(1+s_{1}\right)\left(1+s_{1}^{\mu}\right)}
$$$$
\times \int_{0}^{s_{1}} \frac{\left(s_{1}-s\right)^{\alpha-1}}{\Gamma(\alpha)} q(s) f\left(s, x(s),{ }^{c} D_{0^{+}}^{p} x(s)\right) d s
$$$$
-\frac{s_{2}^{\sigma-\alpha-l}}{\left(1+s_{2}\right)\left(1+s_{2}^{\mu}\right)}
$$$$
\times \int_{0}^{s_{2}} \frac{\left(s_{2}-s\right)^{\alpha-1}}{\Gamma(\alpha)} q(s) f\left(s, x(s),{ }^{c} D_{0^{+}}^{p} x(s)\right) d s \mid
$$$$
+\left|x_{0}\right|\left|\frac{s_{1}^{\sigma-\alpha-l}}{\left(1+s_{1}\right)\left(1+s_{1}^{\mu}\right)}-\frac{s_{2}^{\sigma-\alpha-l}}{\left(1+s_{2}\right)\left(1+s_{2}^{\mu}\right)}\right|
$$$$
+\left|\frac{s_{1}^{\sigma-\alpha-l}}{\left(1+s_{1}\right)\left(1+s_{1}^{\mu}\right)}-\frac{s_{2}^{\sigma-\alpha-l}}{\left(1+s_{2}\right)\left(1+s_{2}^{\mu}\right)}\right| \sum_{j=1}^{\infty} M_{r k}
$$$$
+\widetilde{M}_{r}\left|\frac{s_{1}^{\sigma-\alpha-l}}{\left(1+s_{1}\right)\left(1+s_{1}^{\mu}\right)}-\frac{s_{2}^{\sigma-\alpha-l}}{\left(1+s_{2}\right)\left(1+s_{2}^{\mu}\right)}\right|
$$$$
\times \sum_{j=1}^{k} t_{k}^{\alpha+l} \int_{0}^{1} \frac{(1-w)^{\alpha-1}}{\Gamma(\alpha)} w^{l} d w
$$

$\longrightarrow 0$

uniformly as $s_{1} \longrightarrow s_{2}$ with $s_{1}, s_{2} \in[a, b] \subset\left(t_{k}, t_{k+1}\right]$.

It follows that

$$
\begin{aligned}
& \mid \frac{s_{1}^{\sigma-\alpha-l}}{\left(1+s_{1}\right)\left(1+s_{1}^{\mu}\right)}\left(T_{m} x\right)\left(s_{1}\right) \\
& -\frac{s_{2}^{\sigma-\alpha-l}}{\left(1+s_{2}\right)\left(1+s_{2}^{\mu}\right)}\left(T_{m} x\right)\left(s_{2}\right) \mid \longrightarrow 0
\end{aligned}
$$


uniformly as $s_{1} \rightarrow s_{2}$ with $s_{1}, s_{2} \in[a, b] \subset\left(t_{k}, t_{k+1}\right]$.

In a similar manner, one can find that

$$
\begin{gathered}
\left|\frac{s_{1}^{p+\sigma-\alpha-l}}{1+s_{1}^{\mu}}{ }^{c} D_{t_{k}^{+}}^{p}\left(T_{m} x\right)\left(s_{1}\right)-\frac{s_{2}^{p+\sigma-\alpha-l}}{1+s_{2}^{\mu}}{ }^{c} D_{t_{k}^{+}}^{p}\left(T_{m} x\right)\left(s_{2}\right)\right| \\
=\mid \frac{s_{1}^{p+\sigma-\alpha-l}}{1+s_{1}^{\mu}} \int_{t_{k}}^{s_{1}} \frac{\left(s_{1}-s\right)^{\alpha-p-1}}{\Gamma(\alpha-p)} q(s) \\
\quad \times f\left(s, x(s),{ }^{c} D_{t_{k}^{+}}^{p} x(s)\right) d s \\
\quad-\frac{s_{2}^{p+\sigma-\alpha-l}}{1+s_{2}^{\mu}} \\
\quad \times \int_{t_{k}}^{s_{2}} \frac{\left(s_{2}-s\right)^{\alpha-p-1}}{\Gamma(\alpha-p)} q(s) \\
\quad \times f\left(s, x(s),{ }^{c} D_{t_{k}^{+}}^{p} x(s)\right) d s \mid \\
\longrightarrow 0
\end{gathered}
$$

uniformly as $s_{1} \longrightarrow s_{2}$ with $s_{1}, s_{2} \in[a, b] \subset\left(t_{k}, t_{k+1}\right]$.

From (116) and (117), we deduce that $T_{m} W$ is equicontinuous on finite closed interval on $\left(t_{k}, t_{k+1}\right]$.

Step 4 . We prove that $T_{m} W$ is equiconvergent as $t \rightarrow t_{k}^{+}(k=$ $0,1,2, \ldots)$.

As in Lemma 10, $T_{m} W$ is equiconvergent as $t \rightarrow 0^{+}$. For $t \rightarrow t_{k}^{+}$, we have

$$
\begin{aligned}
& \frac{t^{\sigma-\alpha-l}}{(1+t)\left(1+t^{\mu}\right)} \\
& \times \mid\left(T_{m} x\right)(t)-\left(\sum_{j=1}^{k} \int_{t_{j-1}}^{t_{j}} \frac{\left(t_{j}-s\right)^{\alpha-1}}{\Gamma(\alpha)} q(s)\right. \\
& \quad \times f\left(s, x(s),{ }^{c} D_{t_{k}^{+}}^{p} x(s)\right) d s \\
& \left.\quad+x_{0}+\sum_{j=1}^{k} I_{j}\left(t_{j}, x\left(t_{j}\right)\right)\right) \mid \\
& \leq \frac{t^{\sigma-\alpha-l}}{(1+t)\left(1+t^{\mu}\right)} \quad \\
& \quad \times \int_{t_{k}}^{t} \frac{(t-s)^{\alpha-1}}{\Gamma(\alpha)}\left|q(s) f\left(s, x(s),{ }^{c} D_{t_{k}^{+}}^{p} x(s)\right)\right| d s \\
& \leq M_{r} \frac{t^{\sigma}}{(1+t)\left(1+t^{\mu}\right)} \int_{t_{k} / t}^{1} \frac{(1-w)^{\alpha-1}}{\Gamma(\alpha)} w^{l} d w, \\
& \frac{t^{p+\sigma-\alpha-l}}{1+t^{\mu}} \mid{ }^{c} D_{t_{k}^{+}}\left(T_{m} x\right)(t) \\
& -\int_{t_{k}}^{t} \frac{(t-s)^{\alpha-p-1}}{\Gamma(\alpha-p)} q(s)
\end{aligned}
$$

$$
\begin{array}{r}
\times f\left(s, x(s),{ }^{c} D_{t_{k}^{+}}^{p} x(s)\right) d s \mid \\
\leq M_{r} \frac{t^{\sigma}}{1+t^{\mu}} \int_{t_{k} / t}^{1} \frac{(1-w)^{\alpha-p-1}}{\Gamma(\alpha-p)} w^{l} d w .
\end{array}
$$

Hence, $T_{m} W$ is equiconvergent as $t \rightarrow t_{k}^{+}(k=1,2,3, \ldots)$.

Step 5. $T_{m} W$ is equiconvergent as $t \rightarrow \infty$. Notice that

$$
\begin{aligned}
& \frac{t^{\sigma-\alpha-l}}{(1+t)\left(1+t^{\mu}\right)} \\
& \times \mid\left(T_{m} x\right)(t) \\
& \quad-\left(x_{0}+\sum_{j=1}^{\infty} I_{j}\left(t_{j}, x\left(t_{j}\right)\right)\right. \\
& \quad+\sum_{j=1}^{\infty} \int_{t_{j-1}}^{t_{j}} \frac{\left(t_{j}-s\right)^{\alpha-1}}{\Gamma(\alpha)} q(s)
\end{aligned}
$$$$
\left.\times f\left(s, x(s),{ }^{c} D_{t_{k}^{+}}^{p} x(s)\right) d s\right)
$$

$$
\begin{aligned}
\leq & \frac{t^{\sigma-\alpha-l}}{(1+t)\left(1+t^{\mu}\right)} \\
& \times \int_{t_{k}}^{t} \frac{(t-s)^{\alpha-1}}{\Gamma(\alpha)}\left|q(s) f\left(s, x(s),{ }^{c} D_{t_{k}^{+}}^{p} x(s)\right)\right| d s \\
& +\frac{t^{\sigma-\alpha-l}}{(1+t)\left(1+t^{\mu}\right)} \\
& \times \sum_{j=k+1}^{\infty} \int_{t_{j-1}}^{t_{j}} \frac{\left(t_{j}-s\right)^{\alpha-1}}{\Gamma(\alpha)} \\
\leq & \widetilde{M}_{r} \frac{t^{\sigma}}{(1+t)\left(1+t^{\mu}\right)} \int_{t_{k} / t}^{1} \frac{(1-w)^{\alpha-1}}{\Gamma(\alpha)} w^{l} d w \\
& +\widetilde{M}_{r} \frac{t^{\sigma-\alpha-l}}{(1+t)\left(1+t^{\mu}\right)} \\
& \times \sum_{j=k+1}^{\infty} t_{j}^{\alpha+l} \int_{t_{j-1} / t_{j}}^{1} \frac{(1-w)^{\alpha-1}}{\Gamma(\alpha)} w^{l} d w \\
\leq & \widetilde{M}_{r} M_{\sigma, \mu} \frac{1}{1+t} \frac{\mathbf{B}(\alpha, l+1)}{\Gamma(\alpha)} \\
+ & \widetilde{M}_{r} \frac{1}{\lambda_{0}^{\mu-\sigma+1}} \sum_{j=k+1}^{\infty} \frac{1}{j^{\mu-\sigma+1}} \frac{\mathbf{B}(\alpha, l+1)}{\Gamma(\alpha)} \\
\longrightarrow & \text { uniformly in } W \text { as } t \longrightarrow \infty(k \rightarrow \infty),
\end{aligned}
$$




$$
\begin{aligned}
& \frac{t^{p+\sigma-\alpha-l}}{1+t^{\mu}}\left|D^{c} D_{t_{k}^{+}}^{p}\left(T_{m} x\right)(t)\right| \\
& \leq \frac{t^{p+\sigma-\alpha-l}}{1+t^{\mu}} t^{\alpha+l-p} \\
& \quad \times \int_{t_{k} / t}^{1} \frac{(1-w)^{\alpha-p-1}}{\Gamma(\alpha)} \widetilde{M}_{r} w^{l} d w \\
& \leq \frac{t^{\sigma}}{1+t^{\mu}} \int_{0}^{1} \frac{(1-w)^{\alpha-p-1}}{\Gamma(\alpha)} \widetilde{M}_{r} w^{l} d w \\
& \longrightarrow 0 \quad \text { uniformly in } W \text { as } t \longrightarrow \infty .
\end{aligned}
$$

Hence, $T_{m} W$ is equiconvergent as $t \rightarrow \infty$. This completes the proof in which $T_{m}$ is completely continuous.

Theorem 15. Assume that $\left(H_{1}\right)$ and $\left(H_{2}\right)$ hold. Then IVP (7) has at least one solution $x \in X$ if

$$
\begin{gathered}
\delta_{m}<1 \quad \text { or } \delta_{m}=1 \quad \text { with } N_{0}<1 \quad \text { or } \\
\delta_{m}>1 \quad \text { with } \frac{\|\Psi\|^{1-\delta_{m}}\left(\delta_{m}-1\right)^{\delta_{m}-1}}{\delta_{m}^{\delta_{m}}} \geq N_{0},
\end{gathered}
$$

where $N_{0}=\max \left\{M_{2}, M_{3}\right\}, M_{2}$ is given by (83) and

$$
\begin{aligned}
M_{3}=\sum_{i=1}^{m}\left[\left(M_{\sigma, \mu} \frac{\mathbf{B}(\alpha, l+1)}{\Gamma(\alpha)}\right.\right. & \\
& \left.+\frac{1}{\lambda_{0}^{\mu-\sigma+1}} \frac{\mathbf{B}(\alpha, l+1)}{\Gamma(\alpha)} \sum_{j=1}^{\infty} \frac{1}{j^{\mu-\sigma+1}}\right) \\
& \times\left[A_{i}+B_{i}\right] \\
& \left.+M_{\sigma-\alpha-l, \mu} \sum_{j=1}^{\infty} A_{j i}\right]\|\Psi\|^{\delta_{i}-\delta_{m}} .
\end{aligned}
$$

Proof. Let $Y$ denote the Banach space equipped with the norm $\|\cdot\|$ (introduced in Section 2). Let $T_{m}: Y \rightarrow Y$ be an operator defined by (98). In view of Lemma 8 , we need to show that the operator $T_{m}$ has a fixed point in $Y$ which will be a solution of IVP (7). By Lemma 14, $T_{m}$ is well defined and completely continuous. Lets us introduce

$$
\begin{aligned}
\Phi(t)= & C \int_{t_{k}}^{t} \frac{(t-s)^{\alpha-1}}{\Gamma(\alpha)} q(s) d s \\
& +C \sum_{j=1}^{k} \int_{t_{j-1}}^{t_{j}} \frac{\left(t_{j}-s\right)^{\alpha-1}}{\Gamma(\alpha)} q(s) d s+x_{0} \\
& +\sum_{j=1}^{k} D_{j}, \quad t \in\left(t_{k}, t_{k+1}\right], k=0,1, \ldots
\end{aligned}
$$

It is easy to show that $\Phi \in Y$. Let $\bar{r}>0$ and define

$$
\bar{\Omega}_{\bar{r}}=\{x \in Y:\|x-\Phi\| \leq \bar{r}\} .
$$

For $x \in \bar{\Omega}_{\bar{r}}$, we have $\|x-\Phi\| \leq \bar{r}$. Then

$$
\begin{aligned}
&\|x\|=\max \left\{\sup _{t \in(0, \infty)} \frac{t^{\sigma-\alpha-l}}{(1+t)\left(1+t^{\mu}\right)}|x(t)|,\right. \\
&\left.\sup _{k=0,1,2, \ldots} \sup _{t\left(t_{k}, t_{k+1}\right]} \frac{t^{p+\sigma-\alpha-l}}{1+t^{\mu}}\left|{ }^{c} D_{t_{k}^{+}}^{p} x(t)\right|\right\} \\
& \leq\|x-\Phi\|+\|\Phi\| \leq r+\|\Phi\| .
\end{aligned}
$$

Using the assumptions $\left(H_{1}\right)$ and $\left(H_{2}\right)$, we find that

$$
\begin{aligned}
& \frac{t^{\sigma-\alpha-l}}{(1+t)\left(1+t^{\mu}\right)}\left|\left(T_{m} x\right)(t)-\Phi(t)\right| \\
& \leq \frac{t^{\sigma-\alpha-l}}{(1+t)\left(1+t^{\mu}\right)} \\
& \times \int_{t_{k}}^{t} \frac{(t-s)^{\alpha-1}}{\Gamma(\alpha)}|q(s)| \\
& \times\left|f\left(s, x(s),{ }^{c} D_{0^{+}}^{p} x(s)\right)-C\right| d s \\
& +\frac{t^{\sigma-\alpha-l}}{(1+t)\left(1+t^{\mu}\right)} \\
& \times \sum_{j=1}^{k} \int_{t_{j-1}}^{t_{j}} \frac{\left(t_{j}-s\right)^{\alpha-1}}{\Gamma(\alpha)}|q(s)| \\
& \times\left|f\left(s, x(s),{ }^{c} D_{0^{+}}^{p} x(s)\right)-C\right| d s \\
& +\frac{t^{\sigma-\alpha-l}}{(1+t)\left(1+t^{\mu}\right)} \sum_{j=1}^{k}\left|I_{j}\left(t_{j}, x\left(t_{j}\right)\right)-D_{j}\right| \\
& \leq \frac{t^{\sigma-\alpha-l}}{(1+t)\left(1+t^{\mu}\right)} \\
& \times \int_{t_{k}}^{t} \frac{(t-s)^{\alpha-1}}{\Gamma(\alpha)} s^{l} \\
& \times\left[\sum_{i=1}^{m} A_{i}\left|\frac{t^{\sigma-\alpha-l}}{(1+t)\left(1+t^{\mu}\right)} x(s)\right|^{\delta_{i}}\right. \\
& \left.+\sum_{i=1}^{m} B_{i}\left|\frac{t^{p+\sigma-\alpha-l}}{1+t^{\mu}}{ }^{c} D_{0^{+}}^{p} x(s)\right|^{\delta_{i}}\right] d s \\
& +\frac{t^{\sigma-\alpha-l}}{(1+t)\left(1+t^{\mu}\right)} \\
& \times \sum_{j=1}^{k} \int_{t_{j-1}}^{t_{j}} \frac{\left(t_{j}-s\right)^{\alpha-1}}{\Gamma(\alpha)} s^{l}
\end{aligned}
$$




$$
\begin{aligned}
& \times\left[\sum_{i=1}^{m} A_{i}\left|\frac{t^{\sigma-\alpha-l}}{(1+t)\left(1+t^{\mu}\right)} x(s)\right|^{\delta_{i}}\right. \\
& \left.+\sum_{i=1}^{m} B_{i}\left|\frac{t^{p+\sigma-\alpha-l}}{1+t^{\mu}}{ }^{c} D_{0^{+}}^{p} x(s)\right|^{\delta_{i}}\right] d s \\
& +\frac{t^{\sigma-\alpha-l}}{(1+t)\left(1+t^{\mu}\right)} \\
& \times \sum_{j=1}^{k} \sum_{i=1}^{m} A_{j i}\left|\frac{t_{j}^{\sigma-\alpha-l}}{\left(1+t_{j}\right)\left(1+t_{j}^{\mu}\right)} x\left(t_{j}\right)\right|^{\delta_{i}} \\
& \leq \frac{t^{\sigma-\alpha-l}}{(1+t)\left(1+t^{\mu}\right)} \int_{t_{k}}^{t} \frac{(t-s)^{\alpha-1}}{\Gamma(\alpha)} s^{l} d s \\
& \times \sum_{i=1}^{m}\left[A_{i}+B_{i}\right]\|x\|^{\delta_{i}} \\
& +\frac{t^{\sigma-\alpha-l}}{(1+t)\left(1+t^{\mu}\right)} \sum_{j=1}^{k} \int_{t_{j-1}}^{t_{j}} \frac{\left(t_{j}-s\right)^{\alpha-1}}{\Gamma(\alpha)} s^{l} d s \\
& \times \sum_{i=1}^{m}\left[A_{i}+B_{i}\right]\|x\|^{\delta_{i}} \\
& +\frac{t^{\sigma-\alpha-l}}{(1+t)\left(1+t^{\mu}\right)} \sum_{j=1}^{k} \sum_{i=1}^{m} A_{j i}\|x\|^{\delta_{i}} \\
& \leq \frac{t^{\sigma}}{(1+t)\left(1+t^{\mu}\right)} \int_{t_{k} / t}^{1} \frac{(1-w)^{\alpha-1}}{\Gamma(\alpha)} w^{l} d w \\
& \times \sum_{i=1}^{m}\left[A_{i}+B_{i}\right]\|x\|^{\delta_{i}} \\
& +\frac{t^{\sigma-\alpha-l}}{(1+t)\left(1+t^{\mu}\right)} \\
& \times \sum_{j=1}^{k} t_{j}^{\alpha+l} \int_{t_{j-1} / t_{j}}^{1} \frac{(1-w)^{\alpha-1}}{\Gamma(\alpha)} w^{l} d w \\
& \times \sum_{i=1}^{m}\left[A_{i}+B_{i}\right]\|x\|^{\delta_{i}} \\
& +M_{\sigma-\alpha-l, \mu} \sum_{i=1}^{m} \sum_{j=1}^{\infty} A_{j i}\|x\|^{\delta_{i}} \\
& \leq M_{\sigma, \mu} \int_{0}^{1} \frac{(1-w)^{\alpha-1}}{\Gamma(\alpha)} w^{l} d w \\
& \times \sum_{i=1}^{m}\left[A_{i}+B_{i}\right]\|x\|^{\delta_{i}} \\
& +\frac{t^{\sigma-\alpha-l}}{t^{\mu+1}} \sum_{j=1}^{k} t_{j}^{\alpha+l} \int_{t_{j-1} / t_{j}}^{1} \frac{(1-w)^{\alpha-1}}{\Gamma(\alpha)} w^{l} d w
\end{aligned}
$$$$
\times \sum_{i=1}^{m}\left[A_{i}+B_{i}\right]\|x\|^{\delta_{i}}
$$$$
+M_{\sigma-\alpha-l, \mu} \sum_{i=1}^{m} \sum_{j=1}^{\infty} A_{j i}\|x\|^{\delta_{i}}
$$$$
\leq M_{\sigma, \mu} \int_{0}^{1} \frac{(1-w)^{\alpha-1}}{\Gamma(\alpha)} w^{l} d w
$$$$
\begin{aligned}
& \times \sum_{i=1}^{m}\left[A_{i}+B_{i}\right]\|x\|^{\delta_{i}} \\
& +\sum_{j=1}^{k} \frac{1}{t_{j}^{\mu-\sigma+1}} \int_{0}^{1} \frac{(1-w)^{\alpha-1}}{\Gamma(\alpha)} w^{l} d w \\
& \times \sum_{i=1}^{m}\left[A_{i}+B_{i}\right]\|x\|^{\delta_{i}} \\
& +M_{\sigma-\alpha-l, \mu} \sum_{i=1}^{m} \sum_{j=1}^{\infty} A_{j i}\|x\|^{\delta_{i}}
\end{aligned}
$$$$
\leq M_{\sigma, \mu} \frac{\mathbf{B}(\alpha, l+1)}{\Gamma(\alpha)} \sum_{i=1}^{m}\left[A_{i}+B_{i}\right]\|x\|^{\delta_{i}}
$$$$
+\sum_{j=1}^{\infty} \frac{1}{j^{\mu-\sigma+1} \lambda_{0}^{\mu-\sigma+1}} \frac{\mathbf{B}(\alpha, l+1)}{\Gamma(\alpha)}
$$$$
\times \sum_{i=1}^{m}\left[A_{i}+B_{i}\right]\|x\|^{\delta_{i}}
$$$$
+M_{\sigma-\alpha-l, \mu} \sum_{i=1}^{m} \sum_{j=1}^{\infty} A_{j i}\|x\|^{\delta_{i}}
$$$$
=\left[\left(M_{\sigma, \mu} \frac{\mathbf{B}(\alpha, l+1)}{\Gamma(\alpha)}+\frac{1}{\lambda_{0}^{\mu-\sigma+1}} \frac{\mathbf{B}(\alpha, l+1)}{\Gamma(\alpha)} \sum_{j=1}^{\infty} \frac{1}{j^{\mu-\sigma+1}}\right)\right.
$$$$
\left.\times \sum_{i=1}^{m}\left[A_{i}+B_{i}\right]+M_{\sigma-\alpha-l, \mu} \sum_{i=1}^{m} \sum_{j=1}^{\infty} A_{j i}\right]
$$$$
\times[r+\|\Phi\|]^{\delta_{i}}
$$$$
\leq N_{1}[r+\|\Phi\|]^{\delta_{m}} .
$$

Furthermore, we have

$$
\begin{aligned}
& \frac{t^{p+\sigma-\alpha-l}}{1+t^{\mu}}\left|{ }^{c} D_{t_{k}^{+}}^{p}\left(T_{m} x\right)(t)-{ }^{c} D_{t_{k}^{+}}^{p} \Phi(t)\right| \\
& \leq \frac{t^{p+\sigma-\alpha-l}}{1+t^{\mu}} \\
& \quad \times \int_{t_{k}}^{t} \frac{(t-s)^{\alpha-p-1}}{\Gamma(\alpha-p)}|q(s)| \\
& \quad \times\left|f\left(s, x(s),{ }^{c} D_{t_{k}^{+}}^{p} x(s)\right)-C\right| d s
\end{aligned}
$$




$$
\begin{aligned}
\leq & \frac{t^{p+\sigma-\alpha-l}}{1+t^{\mu}} \\
& \times \int_{t_{k}}^{t} \frac{(t-s)^{\alpha-p-1}}{\Gamma(\alpha-p)} s^{l} \\
& \times\left[\sum_{i=1}^{m} A_{i}\left|\frac{t^{\sigma-\alpha-l}}{(1+t)\left(1+t^{\mu}\right)} x(s)\right|^{\delta_{i}}\right. \\
\leq & \frac{t^{p+\sigma-\alpha-l}}{1+t^{\mu}} \\
& \left.\times \int_{0}^{t} \frac{(t-s)^{\alpha-p-1}}{\Gamma(\alpha-p)} s^{l} B_{i}\left|\frac{t^{p+\sigma-\alpha-l}}{1+t^{\mu}}{ }^{c} D_{t_{k}^{+}}^{p} x(s)\right|^{\delta_{i}}\right] d s \\
\leq & M_{\sigma, \mu} \frac{\mathbf{B}(\alpha-p, l+1)}{\Gamma(\alpha-p)} \sum_{i=1}^{m}\left[A_{i}+B_{i}\right][r+\|\Phi\|]^{\delta_{i}} \\
& \times\left[\sum_{i=1}^{m} \frac{\mathbf{B}(\alpha-p, l+1)}{\Gamma(\alpha-p)} \sum_{i=1}^{m}\left[A_{i}+B_{i}\right]\|x\|^{\delta_{i}}\right. \\
\leq & M_{\sigma, \mu} \frac{\mathbf{B}(\alpha-p, l+1)}{\Gamma(\alpha-p)} \\
& \times\left[\sum_{i=1}^{m} A_{i}\|x\|^{\delta_{i}}+\sum_{i=1}^{m} B_{i}^{m} B_{i}\|x\|^{\delta_{i}}\right] d s
\end{aligned}
$$

Thus, it follows that

$$
\left\|T_{m} x-\Phi\right\| \leq[r+\|\Phi\|]^{\delta_{m}} N_{0}
$$

Now we discuss the cases for different values of $\delta_{m}$.

(i) For $\delta_{m}<1$, we can choose $\bar{r}_{0}>0$ sufficiently large so that $\left[\bar{r}_{0}+\|\Phi\|\right]^{\delta_{m}} N_{0}<\bar{r}_{0}$. Let $\Omega_{\bar{r}_{0}}=\left\{x \in Y:\|x\|<\bar{r}_{0}\right\}$. It is easy to show that $T_{m} \bar{\Omega}_{\bar{r}_{0}} \subset \bar{\Omega}_{\bar{r}_{0}}$. Then, the Schauder fixed point theorem implies that the operator $T_{m}$ has a fixed point $x \in \bar{\Omega}_{\bar{r}_{0}}$, which is a bounded solution of IVP (7).

(ii) For $\delta_{m}=1$, we select

$$
\bar{r}_{0} \geq \frac{\|\Psi\| N_{0}}{1-N_{0}}
$$

Let $\Omega_{\bar{r}_{0}}=\left\{x \in Y:\|x\|<\bar{r}_{0}\right\}$. It can easily be shown that $T_{m} \bar{\Omega}_{\bar{r}_{0}} \subset \bar{\Omega}_{\bar{r}_{0}}$. Then, the Schauder fixed point theorem applies and the operator $T_{m}$ has a fixed point $x \in \bar{\Omega}_{\bar{r}_{0}}$, which is a bounded solution of IVP (7). (iii) For $\delta_{m}>1$, we set $\bar{r}=\bar{r}_{0}=\|\Phi\| /\left(\delta_{m}-1\right)$ so that

$$
\frac{\bar{r}_{0}}{\left(\bar{r}_{0}+\|\Phi\|\right)^{\delta_{m}}}=\frac{\|\Phi\|^{1-\delta_{m}}\left(\delta_{m}-1\right)^{\delta_{m}-1}}{\delta_{m}^{\delta_{m}}} \geq N_{0} .
$$

Let $\Omega_{\bar{r}_{0}}=\left\{x \in Y:\|x\|<\bar{r}_{0}\right\}$. Then we can show that $T_{m} \bar{\Omega}_{r_{0}} \subset$ $\bar{\Omega}_{r_{0}}$. Thus, by the Schauder fixed point theorem, the operator $T_{m}$ has a fixed point $x \in \bar{\Omega}_{\bar{r}_{0}}$, which is a solution of IVP (7). This completes the proof.

Theorem 16. Suppose that $\left(H_{1}\right)$ and $\left(H_{2}\right)$ hold with $\delta_{m}=1$. Then IVP (7) has a unique solution $x \in Y$ if $N_{0}<1$.

Proof. The proof is similar to that of Theorem 12, so we omit it.

\section{Applications}

Malthusian geometrical law is expressed as $N^{\prime}(t)=r N(t)$, where $N(t)$ is the population at time $t$ and $r$ is the proportionality constant. When the growth of the population in any environment is stopped due to the density of the population, this model modifies to a nonlinear logistic model of the form $N^{\prime}(t)=r N(t)(1-N(t) / \pi)$. The generalization of the nonlinear logistic model is represented by $N^{\prime}(t)=$ $r N(t)\left[1-(N(t) / \pi)^{\alpha}\right] / \alpha$. For $\alpha \rightarrow 0$, the model is known as the Gompertz model and can be found in the literature on actuarial science and mortality analysis of elderly person [31].

In [32], Das et al. presented the following fractional-order logistic model (Das Model):

$$
D_{0^{+}}^{\beta} N(t)=\frac{r}{\alpha} N(t)\left[1-\left(\frac{N(t)}{\pi}\right)^{\alpha}\right], \quad 0<\beta \leq 1 .
$$

In [33], the authors presented the following logistic model with fractional order:

$$
\begin{gathered}
{ }^{c} D_{0^{+}}^{\alpha} x(t)=x(t)[a(t)-b(t)(x(t))], \quad t \in(0, \infty), t \neq t_{k}, \\
\Delta x\left(t_{k}\right)=I_{k}\left(x\left(t_{k}^{-}\right)\right), \quad k=1,2, \ldots, \\
x(0)=x_{0},
\end{gathered}
$$

where $T>0$ is a constant, $I_{k}: R \rightarrow R(k=1,2, \ldots, m)$ are impulse functions, $a(t) \in\left[a_{*}, a^{*}\right]$, and $b(t) \in\left[b_{*}, b^{*}\right]$ with $a_{*}>0, b_{*}>0$. 
As an application of the main results established in the paper, we discuss the sufficient conditions for the existence and asymptotic behavior of solutions for the logistic models:

$$
\begin{gathered}
{ }^{c} D_{0^{+}}^{\alpha} x(t)=x(t)\left[a(t)-b(t)(x(t))^{\delta}\right], \quad t \in(0, \infty), t \neq t_{k}, \\
\Delta x\left(t_{k}\right)=I_{k}\left(t_{k}, x\left(t_{k}\right)\right), \quad k=1,2, \ldots, \\
x(0)=x_{0}, \\
{ }^{c} D_{*}^{\alpha} x(t)=x(t)\left[a(t)-b(t)(x(t))^{\delta}\right], \quad t \in(0, \infty), t \neq t_{k}, \\
\Delta x\left(t_{k}\right)=I_{k}\left(t_{k}, x\left(t_{k}\right)\right), \quad k=1,2, \ldots, \\
x(0)=x_{0},
\end{gathered}
$$

where $0<t_{1}<t_{2}<t_{3}<\cdots, \alpha \in(0,1], \delta>0$, $a, b:(0, \infty) \rightarrow R$ are continuous functions, and $a_{k} \in R \rightarrow$ $R(k=1,2,3, \ldots)$ are constants.

Theorem 17. Suppose that

$$
\begin{aligned}
& \frac{(1+t)\left(1+t^{\mu}\right)}{t^{\sigma-\alpha-l}} a(t) \leq a_{0}, \\
& \left(\frac{(1+t)\left(1+t^{\mu}\right)}{t^{\sigma-\alpha-l}}\right)^{\delta+1} b(t) \leq b_{0}, \\
& t \in(0, \infty),
\end{aligned}
$$

and there exists $D_{k} \in R, A_{k 1}, A_{k 2} \geq 0$ such that

$$
\begin{aligned}
& \left|I_{k}\left(t_{k}, \frac{(1+t)\left(1+t^{\mu}\right)}{t^{\sigma-\alpha-l}} u\right)-D_{k}\right| \\
& \quad \leq A_{k 1}|u|+A_{k 2}|u|^{2}, \quad k=1,2,3, \ldots, u \in R .
\end{aligned}
$$

Then IVP (133) has at least one solution if

$$
4\|\Phi\| M_{0} \leq 1,
$$

where

$$
\begin{gathered}
\Phi(t)=x_{0}+\sum_{j=1}^{k} D_{j}, \quad t \in\left(t_{k}, t_{k+1}\right], k=0,1,2, \ldots, \\
M_{1}=\left[M_{\sigma, \mu} \frac{\mathbf{B}(\alpha, l+1)}{\Gamma(\alpha)} a_{0}+M_{\sigma-\alpha-l, \mu} \sum_{j=1}^{\infty} A_{j 1}\right]\|\Psi\|^{-1} \\
+M_{\sigma, \mu} \frac{\mathbf{B}(\alpha, l+1)}{\Gamma(\alpha)} b_{0}+M_{\sigma-\alpha-l, \mu} \sum_{j=1}^{\infty} A_{j 2}, \\
M_{2}=M_{\sigma, \mu} \frac{B(\alpha-p, l+1)}{\Gamma(\alpha-p)}\left(a_{0}+b_{0}\right), \\
M_{0}=\max \left\{M_{1}, M_{2}\right\} .
\end{gathered}
$$

Proof. Let $f(t, u, v)=u\left[a(t)-b(t) u^{\delta}\right]$. Then

$$
\begin{aligned}
& \left|f\left(t, \frac{(1+t)\left(1+t^{\mu}\right)}{t^{\sigma-\alpha-l}} u, \frac{1+t^{\mu}}{t^{p+\sigma-\alpha-l}}\right)\right| \\
& =\frac{(1+t)\left(1+t^{\mu}\right)}{t^{\sigma-\alpha-l}} a(t)|u| \\
& \quad+b(t)\left(\frac{(1+t)\left(1+t^{\mu}\right)}{t^{\sigma-\alpha-l}}\right)^{\delta+1}|u|^{\delta+1} \\
& \leq a_{0}|u|+b_{0}|u|^{\delta+1} .
\end{aligned}
$$

In association with Theorem 11, we choose $C=0, A_{1}=a_{0}$, $A_{1}=a_{1}, \delta_{1}=1, \delta_{2}=2, B_{1}=B_{2}=0$. Then the conditions $\left(H_{1}\right)$ and $\left(H_{2}\right)$ hold. By Theorem 11, IVP (133) has at least one solution. This completes the proof.

Theorem 18. Suppose that

$$
\begin{gathered}
\frac{(1+t)\left(1+t^{\mu}\right)}{t^{\sigma-\alpha-l}} a(t) \leq a_{0}, \\
\left(\frac{(1+t)\left(1+t^{\mu}\right)}{t^{\sigma-\alpha-l}}\right)^{\delta+1} b(t) \leq b_{0},
\end{gathered}
$$

$$
t \in(0, \infty)
$$

and there exists $D_{k} \in R, A_{k 1}, A_{k 2} \geq 0$ such that

$$
\begin{aligned}
& \left|I_{k}\left(t_{k}, \frac{(1+t)\left(1+t^{\mu}\right)}{t^{\sigma-\alpha-l}} u\right)-D_{k}\right| \\
& \quad \leq A_{k 1}|u|+A_{k 2}|u|^{2}, \quad k=1,2,3, \ldots, u \in R .
\end{aligned}
$$

Then IVP (134) has at least one solution if

$$
4 N_{0}\|\Psi\| \leq 1
$$

where

$$
\Psi(t)=x_{0}+\sum_{j=1}^{k} D_{j}, \quad t \in\left(t_{k}, t_{k+1}\right], \quad k=0,1,2, \ldots
$$




$$
\begin{gathered}
M_{3}=\left[\left(M_{\sigma, \mu} \frac{\mathbf{B}(\alpha, D+1)}{\Gamma(\alpha)}\right.\right. \\
\left.\quad+\frac{1}{\lambda_{0}^{\mu-\sigma+1}} \frac{\mathbf{B}(\alpha, l+1)}{\Gamma(\alpha)} \sum_{j=1}^{\infty} \frac{1}{\mu^{\mu-\sigma+1}}\right) a_{0} \\
\left.+M_{\sigma-\alpha-l, \mu} \sum_{j=1}^{\infty} A_{j 1}\right]\|\Psi\|^{-1} \\
+\left(M_{\sigma, \mu} \frac{\mathbf{B}(\alpha, l+1)}{\Gamma(\alpha)}\right. \\
\left.\quad+\frac{1}{\lambda_{0}^{\mu-\sigma+1}} \frac{\mathbf{B}(\alpha, l+1)}{\Gamma(\alpha)} \sum_{j=1}^{\infty} \frac{1}{j^{\mu-\sigma+1}}\right) b_{0} \\
+M_{\sigma-\alpha-l, \mu} \sum_{j=1}^{\infty} A_{j 2}, \\
M_{2}=M_{\sigma, \mu} \frac{\mathbf{B}(\alpha-p, l+1)}{\Gamma(\alpha-p)}\left(a_{0}+b_{0}\right), \\
N_{0}=\max \left\{M_{2}, M_{3}\right\} .
\end{gathered}
$$

Proof. The proof immediately follows from Theorem 15.

\section{Conflict of Interests}

The authors declare that there is no conflict of interests regarding the publication of this paper.

\section{Acknowledgments}

The research of Bashir Ahmad is partially supported by Deanship of Scientific Research (DSR), King Abdulaziz University, Jeddah, Saudi Arabia. This work was supported by the Natu-ral Science Foundation of Guangdong province (no. S2011010001900) and the Guangdong Higher Education Foundation for High-Level Talents.

\section{References}

[1] V. D. Milman and A. D. Myshkis, "On the stability of motion in the presence of impulses," Siberial Mathematical Journal, vol. 1, no. 2, pp. 233-237, 1960 (Russian).

[2] A. M. Samoilenko and N. A. Perestyuk, Differential Equations with Impulses, Viska Scola, Kiev, Ukraine, 1987.

[3] V. Lakshmikantham, D. Bainov, and P. Simeonov, Theory of Impulsive Differential Equations, World Scientific, Singapore, 1989.

[4] D. D. Baino and P. S. Simeonov, Systems with Impulsive Effects, Horwood, Chichister, UK, 1989.

[5] D. D. Baino and V. Covachev, Differential Equations with a Small Perturbations, World Scientific, New Jersey, NJ, USA, 1994.

[6] M. Benchohra, J. Henderson, and S. Ntonyas, Impulsive Differential Equations and Inclusions, vol. 2, Hindawi Publishing Corporation, New York, NY, USA, 2006.
[7] F. Mainardi, "Fractional Calculus: some basic problems in continuum and statistical mechanics," in Fractals and Fractional Calculus in Continuum Mechanics, A. Carpinteri and F. Mainardi, Eds., Springer, New York, NY, USA, 1997.

[8] R. Hilfer, Applications of Fractional Calculus in Physics, World Scientific, River Edge, NJ, USA, 2000.

[9] A. A. Kilbas, H. M. Srivastava, and J. J. Trujjilo, Theory and Applications of Fractional Differential Equations, vol. 204 of North-Holland Mathematics Studies, Elsevier, London, UK, 2006.

[10] I. Podlubny, , Fractional Differential Equations, Academic Press, London, UK, 1999.

[11] R. P. Agarwal, M. Benchhra, and B. A. Slimani, "Existence results for differential equations with fractional order and impulses," Memoirs on Differential Equations and Mathematical Physics, vol. 44, pp. 1-21, 2008.

[12] J. Henderson and A. Ouahab, "Impulsive differential inclusions with fractional order," Computers and Mathematics with Applications, vol. 59, no. 3, pp. 1191-1226, 2010.

[13] Y. Liu, "Existence and uniqueness of solutions for initial value problems of multi-order fractional differential equations on the half lines," Scientia Sinica Mathematica, vol. 42, no. 7, pp. 735756, 2012 (Chinese).

[14] Y. Liu, "Existence of solutions for impulsive differential models on half lines involving Caputo fractional derivatives," Communications in Nonlinear Science and Numerical Simulation, vol. 18, pp. 2604-2625, 2013.

[15] J. Wang, Y. Yang, and W. Wei, "Nonlocal impulsive problems for fractional differential equationswith time varying generating operators in Banach spaces, Opuscula mathematica," vol. 30, pp. 361-381, 2010.

[16] S. Zhang, "Monotone iterative method for initial value problem involving Riemann-Liouville fractional derivatives," Nonlinear Analysis: Theory, Methods and Applications, vol. 71, no. 5-6, pp. 2087-2093, 2009.

[17] B. Ahmad and J. J. Nieto, "Existence of solutions for impulsive anti-periodic boundary value problems of fractional order," Taiwanese Journal of Mathematics, vol. 15, no. 3, pp. 981-993, 2011.

[18] B. Ahmad and G. Wang, "Impulsive anti-periodic boundary value problem for nonlinear differential equations of fractional order," Computers and Mathematics With Applications, vol. 59, pp. 1341-1349, 2010.

[19] J. Cao and H. Chen, "Impulsive fractional differential equations with nonlinear boundary conditions," Mathematical and Computer Modelling, vol. 55, no. 3-4, pp. 303-311, 2012.

[20] J. Cao and H. Chen, "Some results on impulsive boundary value problem for fractional differentialinclusions," Electronic Journal of Qualitative Theory of Differential Equations, vol. 11, pp. 1-24, 2010.

[21] Y. Tian and Z. Bai, "Existence results for the three-point impulsive boundary value problem involving fractional differential equations," Computers and Mathematics with Applications, vol. 59, no. 8, pp. 2601-2609, 2010.

[22] X. Wang, "Impulsive boundary value problem for nonlinear differential equations of fractional order," Computers and Mathematics with Applications, vol. 62, no. 5, pp. 2383-2391, 2011.

[23] G. Wang, B. Ahmad, and L. Zhang, "Some existence results for impulsive nonlinear fractional differential equations with mixed boundary conditions," Computers and Mathematics with Applications, vol. 62, no. 3, pp. 1389-1397, 2011. 
[24] J. Wang, Y. Zhou, and M. Feckana, “On recent developments in the theory of boundary value problems for impulsive fractional differential equations," Computers and Mathematics With Applications, vol. 64, pp. 3008-3020, 2012.

[25] G. Wang, L. Zhang, and G. Song, "Systems of first order impulsive functional differential equations with deviating arguments and nonlinear boundary conditions," Nonlinear Analysis: Theory, Methods and Applications, vol. 74, no. 3, pp. 974-982, 2011.

[26] L. Yang and H. Chen, "Nonlocal boundary value problem for impulsive differential equations of fractional order," Advances in Difference Equations, vol. 2011, Article ID 404917, 16 pages, 2011.

[27] R. P. Agarwal, M. Benchohra, S. Hamani, and S. Pinelas, "Upper and lower solutions method for impulsive differential equations involving the Caputo fractional derivatives," Memoirs on Differential Equations and Mathematical Physics, vol. 53, pp. $1-12,2011$.

[28] R. P. Agarwal, M. Benchohra, and B. A. Slimani, "Existence results for differential, equations with fractional order and impulses," Memoirs on Differential Equations and Mathematical Physics, vol. 44, pp. 1-21, 2008.

[29] M. Fečkan, Y. Zhou, and J. Wang, "On the concept and existence of solution for impulsive fractional differential equations," Communications in Nonlinear Science and Numerical Simulation, vol. 17, no. 7, pp. 3050-3060, 2012.

[30] Y. Liu and B. Ahmad, "An impulsive booundary value problem for singular multi-term fractional differential equations on the half line," Communications on Applied Nonlinear Analysis, vol. 20, pp. 21-54, 2013.

[31] J. S. Cramer, "The origins and development of the logit model," University of Amsterdam and Tinbergen Institute Manuscript, 2003, http://www.cambridge.org/resources/0521815886/ 1208_default.pdf.

[32] S. Das, P. K. Gupta, and K. Vishal, "Approximate approach to the Das model of fractional logistic population growth," Applications and Applied Mathematics, vol. 5, no. 10, pp. 17021708, 2010.

[33] L. Mahto, S. Abbas, and A. Favini, "Analysis of Caputo impulsive fractional order differential equations with applications," International Journal of Differential Equations, vol. 2013, Article ID 704547, 11 pages, 2013. 


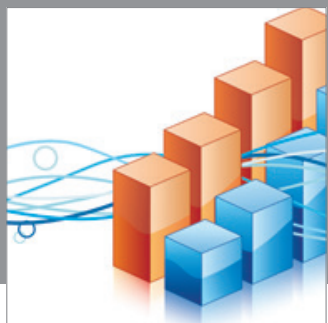

Advances in

Operations Research

mansans

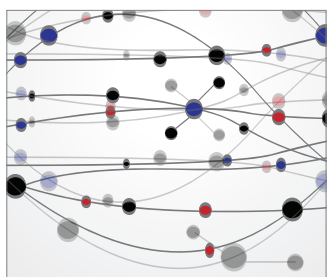

The Scientific World Journal
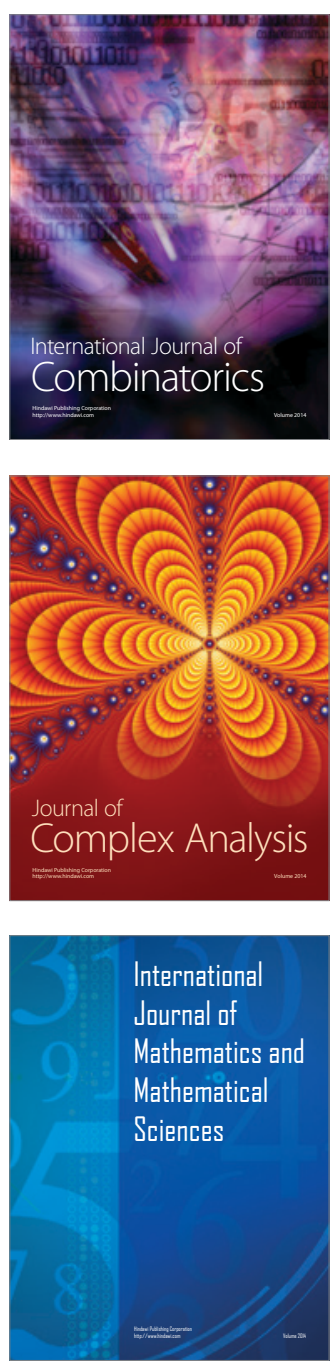
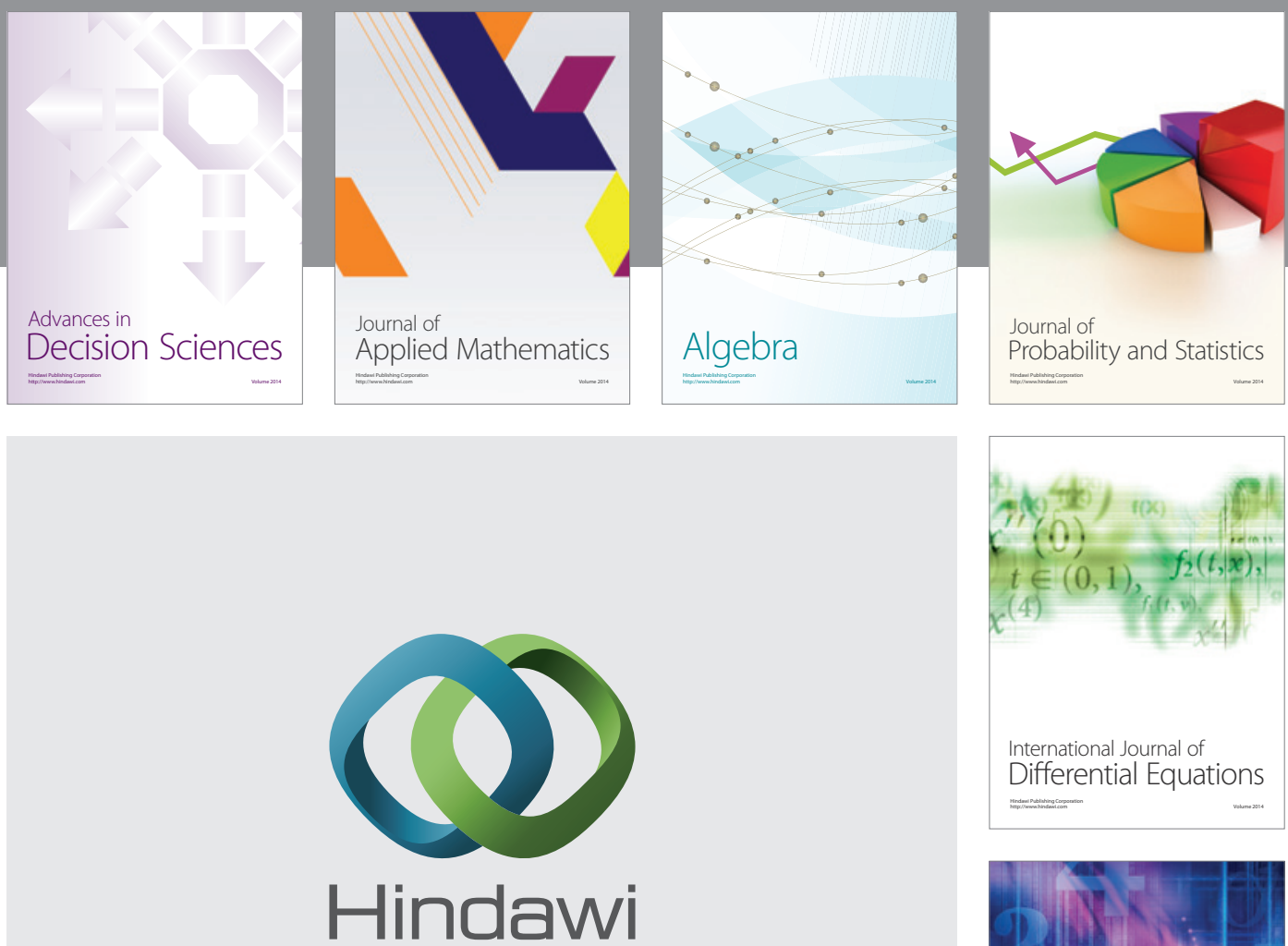

Submit your manuscripts at http://www.hindawi.com
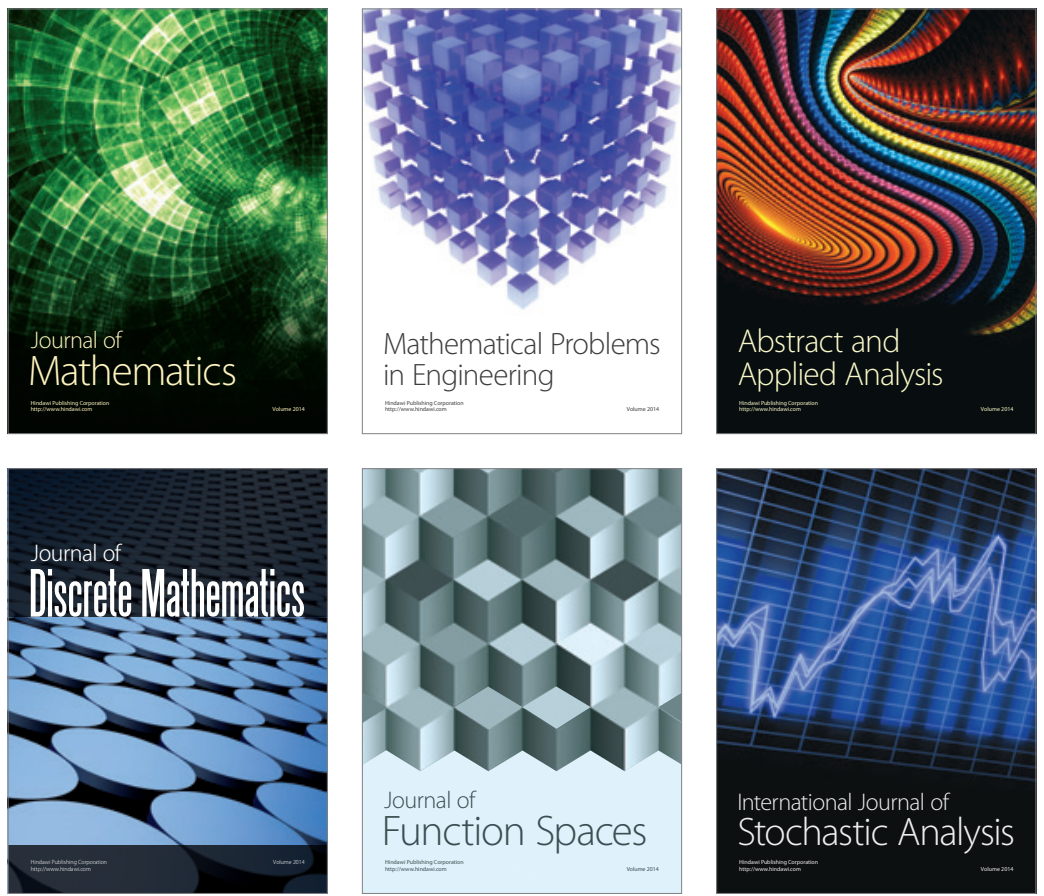

Journal of

Function Spaces

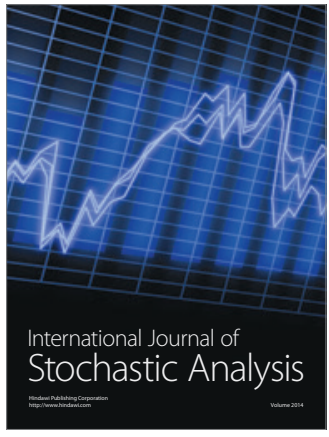

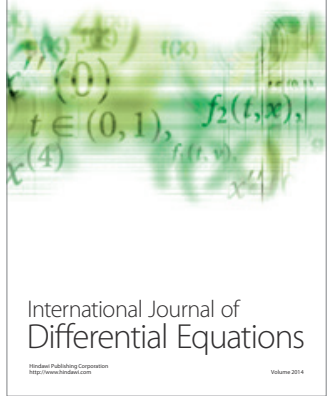
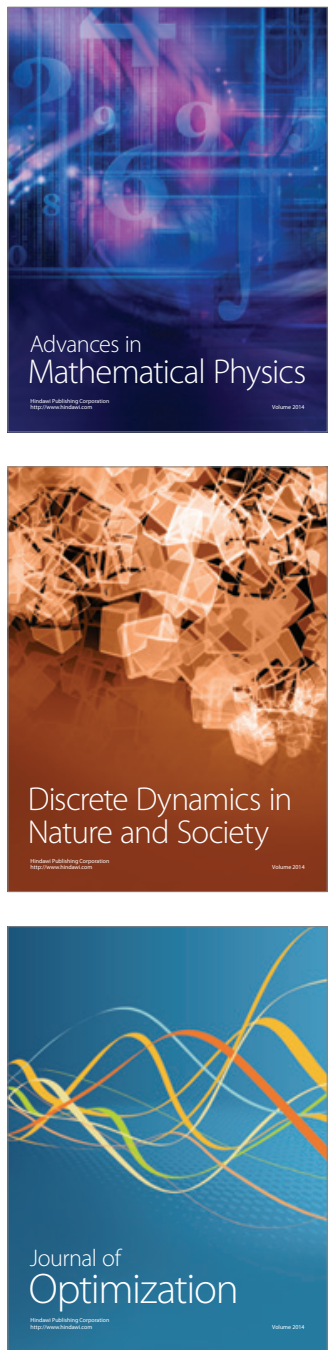